This item was submitted to Loughborough's Research Repository by the author.

Items in Figshare are protected by copyright, with all rights reserved, unless otherwise indicated.

\title{
Influence of the Reynolds number on the instant flow evolution of a turbulent rectangular free jet of air
}

PLEASE CITE THE PUBLISHED VERSION

http://dx.doi.org/10.1016/j.ijheatfluidflow.2014.10.001

PUBLISHER

(C) Elsevier

VERSION

AM (Accepted Manuscript)

\section{PUBLISHER STATEMENT}

This work is made available according to the conditions of the Creative Commons Attribution-NonCommercialNoDerivatives 4.0 International (CC BY-NC-ND 4.0) licence. Full details of this licence are available at: https://creativecommons.org/licenses/by-nc-nd/4.0/

\section{LICENCE}

CC BY-NC-ND 4.0

\section{REPOSITORY RECORD}

Gori, Fabio, Ivano Petracci, and Matteo Angelino. 2017. "Influence of the Reynolds Number on the Instant Flow Evolution of a Turbulent Rectangular Free Jet of Air”. figshare. https://hdl.handle.net/2134/26086. 


\title{
Influence of the Reynolds number on the instant flow evolution of a turbulent rectangular free jet of air
}

\author{
Fabio Gori*, Ivano Petracci, Matteo Angelino \\ University of Rome "Tor Vergata”, Via del Politecnico 1, 00133 Rome, Italy
}

\section{A B S T R A C T}

The paper is aimed at investigating the influence of the Reynolds number on the instant flow evolution of a rectangular free jet of air in the range of Reynolds numbers from $\operatorname{Re}=35,300$ to $\operatorname{Re}=2,200$, where the Reynolds number, Re, is defined according to the hydraulic diameter, $D$, of a rectangular slot of height $H$, equal to about $\mathrm{D}=2 \mathrm{H}$. The Particle Image Velocimetry (PIV) technique allows obtaining the instant PIV visualizations on the central symmetry section of the rectangular jet. The visual inspection of the instant frames with one and two vortices, except for $\operatorname{Re}=35,300$ where only one vortex images are detected, shows that after the jet exit is present the Flow with Constant Instant Height, with a length $L_{C I H}$ which increases with the decrease of the Reynolds number, from a ratio $L_{C I H} / H$ equal to $L_{C I H} / H=0.9$ at $R e=35,300$ to $L_{C I H} / H=4.0$ at $R e=2,200$. The instant PIV measurements, carried out at several distances from the jet exit, show that the variations of the ratio $U / U_{0}$ of the centerline instant velocity, $U$, to the exit average velocity, $U_{0}$, remain below $\pm 4 \%$ for a length $L_{C I V}$, defining the Flow with Constant Instant Velocity on the centerline. The ratio $L_{C I V} / H$ increases from $L_{C I V} / H=1.1$ at $\operatorname{Re}=35,300$ to $L_{C I V} / H=4.1$ at $\operatorname{Re}$ $=2,200$ and is quite similar to $L_{C I H} / H$. The instant PIV measurements of the centerline turbulence inten-sity, $T u$, show that its variations remain below $\pm 4 \%$ for a length $L_{C I T}$, defining the Flow with Constant Instant Turbulence on the centerline. The ratio $L_{C I T} / H$ is equal to $L_{C I V} / H$. The instant PIV velocity profiles in the transverse direction, are practically unchanged for a length $L_{U V P}$ which defines the flow with Unchanged Velocity Profile. The four lengths $L_{U V P}=L_{C I H}=$ $L_{C I V}=L_{C I T}$ are considered equal, defining the Flow with Negligible Disturbances, with length $L_{\mathrm{ND}}$, or first type of flow, $L_{1}$, which is in agreement with the average length of the flow with Constant Height, $L_{C H}$, in the turbulent and laminar regime, and with the average length of the Undisturbed flow, $L_{U}$, in the turbulent regime. The instant PIV frames show the presence, after the flow with negligible disturbances, of the Flow with Small Variations of the Height, where the jet height varies slightly without forming the vortex. The length of the flow with Small Vari-ation of the Height is $L_{S V H}$, and the first vortex appears after the total length $\left(L_{N D}+L_{S V H}\right)$. The variations of the instant PIV measurements of the centerline turbulence intensity, $T u$, increase after the length $L_{C I T}$, but remaining below $\pm 5-9 \%$ for a length $L_{S V T}$, which defines the Flow with Small Variation of the Turbulence. The total length $\left(L_{C I T}+L_{S V T}\right)$ is comparable to $\left(L_{C H}+L_{S V H}\right)$ in the turbulent and laminar regimes, and can be assumed equal to the sum of the lengths of the flows with negligible and small disturbances, $L_{N D}$ $+L_{S D}=L_{2}$, which is in agreement with the average length of the undisturbed flow, $L_{U}$, in the laminar regime. The instant PIV frames show the flow with Coherent Vortices after $L_{2}$, with a length $L_{C V}$, followed by the vortices breakdown. The instant PIV frames allow visualizing the end of the flow with Coherent Vortices, defining the total length of the flows with negligible and small disturbances, and coherent vortices, as $L_{N D}+L_{S D}+L_{C V}=L_{3}$, which is in good agreement with the average length of the potential core, $L_{P}$.

\section{Introduction}

The evolution of turbulent free jets has been investigated at a great extent in the last decades. The amount of papers dealing with rectangular and round jets is so vast that it is impossible to mention all of them, and only those pertaining to rectangular free jets are therefore mentioned in the present paper.

The flow evolution of a jet, emerging from a two-dimensional rectangular slot without converging duct, was proposed by Albertson et al. (1948), and an updated version is reported in Gori et al. (2013). The jet interacts with the stagnant fluid just after 


\begin{tabular}{|c|c|c|c|}
\hline \multicolumn{4}{|c|}{ Nomenclature } \\
\hline$B$ & bias & $L_{3}$ & length of the flow with negligible, small dis- \\
\hline d & $\begin{array}{l}\text { reference distance in the calibration board } \\
\text { (m) }\end{array}$ & & $\begin{array}{l}\text { turbances and coherent vortices (instant) } \\
(\mathrm{m})\end{array}$ \\
\hline$D$ & hydraulic diameter of the slot (m) & $L_{C H}$ & length of the flow with constant height \\
\hline$D^{\prime}$ & reference distance in the image plane (pix- & & (average) $(\mathrm{m})$ \\
\hline$F_{3}$ & $\begin{array}{l}\text { els) } \\
\text { flow with constant instant height }\end{array}$ & $L_{C T}$ & length of the flow with constant turbulence \\
\hline $\mathrm{F}_{\mathrm{CIV}}$ & $\begin{array}{l}\text { flow with constant instant velocity on the } \\
\text { centerline }\end{array}$ & $\begin{array}{l}L_{P} \\
L_{S}\end{array}$ & $\begin{array}{l}\text { length of the potential core (average) }(\mathrm{m}) \\
\text { length of the hypothetical line-source }(\mathrm{m})\end{array}$ \\
\hline $\mathrm{F}_{\mathrm{CIT}}$ & $\begin{array}{l}\text { flow with constant instant turbulence on the } \\
\text { centerline }\end{array}$ & $\begin{array}{l}L_{S} \\
M\end{array}$ & $\begin{array}{l}\text { length of the undisturbed flow (average) (m) } \\
\text { magnification factor }\end{array}$ \\
\hline $\mathrm{F}_{\mathrm{UVP}}$ & flow with unchanged instant velocity profile & $\operatorname{Re}$ & Reynolds number, defined according to $D$ \\
\hline $\mathrm{F}_{\mathrm{ND}}$ & flow with Negligible Disturbances (instant) & $\mathrm{Re}_{H}$ & Reynolds number, defined according to $H$ \\
\hline $\mathrm{F}_{\mathrm{SVH}}$ & $\begin{array}{l}\text { flow with small instant variation of the } \\
\text { height }\end{array}$ & $\begin{array}{l}S \\
t\end{array}$ & $\begin{array}{l}\text { average precision index } \\
\text { time }(s)\end{array}$ \\
\hline $\mathrm{F}_{\mathrm{SVT}}$ & $\begin{array}{l}\text { flow with small instant variation of the } \\
\text { turbulence }\end{array}$ & $\begin{array}{l}T u[\%]=\operatorname{RMS}\left(U^{\prime}\right) / \bar{U}_{0} \\
U\end{array}$ & $\begin{array}{l}\text { turbulence intensity } \\
\text { instant velocity in } x \text {-direction }\left(\mathrm{m} \mathrm{s}^{-1}\right)\end{array}$ \\
\hline $\mathrm{F}_{\mathrm{CH}}$ & flow with constant height (average) & $U^{\prime}$ & time fluctuation of velocity in $x$-direction \\
\hline $\mathrm{F}_{\mathrm{CT}}$ & $\begin{array}{l}\text { flow with constant turbulence on the } \\
\text { centerline (average) }\end{array}$ & $\bar{U}$ & $\begin{array}{l}\left(\mathrm{m} \mathrm{s}^{-1}\right) \\
\text { time average velocity in } x \text {-direction }\left(\mathrm{m} \mathrm{s}^{-1}\right)\end{array}$ \\
\hline $\begin{array}{l}\mathrm{F}_{\mathrm{U}} \\
H\end{array}$ & $\begin{array}{l}\text { undisturbed flow (average) } \\
\text { height of the rectangular slot (m) }\end{array}$ & $U_{a v}$ & $\begin{array}{l}\text { time average velocity in the cross section } \\
\left(\mathrm{m} \mathrm{s}^{-1}\right)\end{array}$ \\
\hline$L_{C I H}$ & $\begin{array}{l}\text { length of the flow with constant instant } \\
\text { height }(\mathrm{m})\end{array}$ & $U_{0}$ & $\begin{array}{l}\text { instant velocity on centerline of the slot exit } \\
\left(\mathrm{m} \mathrm{s}^{-1}\right)\end{array}$ \\
\hline$L_{C I T}$ & $\begin{array}{l}\text { length of the flow with constant instant } \\
\text { turbulence on the centerline }(\mathrm{m})\end{array}$ & $\bar{U}_{0}$ & $\begin{array}{l}\text { time average velocity on centerline of the } \\
\text { slot exit }\left(\mathrm{m} \mathrm{s}^{-1}\right)\end{array}$ \\
\hline$L_{C I V}$ & $\begin{array}{l}\text { length of the flow with constant instant } \\
\text { velocity on the centerline }(\mathrm{m})\end{array}$ & $\begin{array}{l}\dot{V} \\
W\end{array}$ & $\begin{array}{l}\text { volume flow rate }\left(\mathrm{m}^{3} / \mathrm{s}\right) \\
\text { width of the slot }(\mathrm{m})\end{array}$ \\
\hline$L_{U V P}$ & $\begin{array}{l}\text { length of the flow with unchanged instant } \\
\text { velocity profile }(m)\end{array}$ & $\begin{array}{l}x \\
X\end{array}$ & $\begin{array}{l}\text { stream-wise distance from the slot exit }(\mathrm{m}) \\
\text { general physical quantity }(\cdot)\end{array}$ \\
\hline$L_{N D}$ & $\begin{array}{l}\text { length of the flow with negligible } \\
\text { disturbances (instant) }(\mathrm{m})\end{array}$ & $y$ & transverse distance from the jet axis (m) \\
\hline$L_{S V H}$ & $\begin{array}{l}\text { length of the flow with small instant } \\
\text { variation of height }(\mathrm{m})\end{array}$ & Greek & narticles disnlacement (nixels) \\
\hline$L_{S V T}$ & $\begin{array}{l}\text { length of the flow with small instant } \\
\text { variation of the turbulence (m) }\end{array}$ & $\vartheta_{\vartheta}$ & $\begin{array}{l}\text { angle between light sheet and calibration } \\
\text { board (rad) }\end{array}$ \\
\hline$L_{1}$ & $\begin{array}{l}\text { length of the flow with negligible distur- } \\
\text { bances (instant })(\mathrm{m})\end{array}$ & $v$ & kinematics viscosity of air $\left(\mathrm{m} \mathrm{s}^{-2}\right)$ \\
\hline$L_{2}$ & $\begin{array}{l}\text { length of the flow with negligible and small } \\
\text { disturbances (instant) }(\mathrm{m})\end{array}$ & & \\
\hline
\end{tabular}

the exit, and two zones are defined in comparison to the length of the potential core, $L_{P}$. The first one, for distances smaller than $L_{P}$, is the zone of flow establishment and the second one, for distances greater than $L_{P}$, is the zone of established flow or fully developed region. Moreover, two regions of flow are identified in the zone of flow establishment: the region of mixing fluid, at the border with the stagnant fluid, and the potential core, where the average velocity on the centerline maintains equal to the exit one.

The origin of the zone of established flow, suggested by Albertson et al. (1948), is indicated by the distance of the hypothetical line-source from the slot exit, $L_{s}$. Van der Hegge Zijnen (1958) performed average velocity measurements at $\mathrm{Re}_{H}=13,300$ with two types of rectangular slits and found two different values of the length of the hypothetical line source, $L_{S}=-0.6 \mathrm{H}$ and $L_{S}=-1.7 \mathrm{H}$, where a negative sign means that the line source is upstream the slot exit. As far as the distance of the line source is concerned, Bradbury (1965) found a length $L_{S}=-3 H$, while Gutmark and Wygnanski (1976) measured a length $L_{S}=-2 H$ at $\mathrm{Re}_{H}=30,000$. The deformation of a three-dimensional rectangular jet was investigated by Abramovich (1982) who declared that the line-source of the jet was upstream the slot exit at $L_{S}=-4.5 \mathrm{H}$, independently from the Reynolds number.
Preliminary average velocity and turbulence measurements with the Hot Wire Anemometer (HWA) and shadowgraph visualizations were carried out by Gori et al. (2002a) in an air jet emerging from a rectangular slot of height $H=4.25 \mathrm{~mm}$ and width $W=106 \mathrm{~mm}$, after a small wind tunnel, called WT1. The main conclusion of the HWA measurements at $\operatorname{Re}=11,300$ was the observation that just after the exit, and for a length of about $L_{U}=1.6 \mathrm{H}$, is present a type of flow where velocity and turbulence remain unchanged, compared to those measured on the exit, and was called Undisturbed Region of Flow. The shadowgraph visualizations at $\operatorname{Re}=10,000$ showed the presence of a flow with Constant Height, with a length $L_{C H}$ almost equal to that of the Undisturbed Region of Flow, $L_{U}$.

Average fluid dynamics measurements of velocity and turbulence were carried out by Gori and Petracci (2003b) with a different small wind tunnel, called WT2, equipped with a rectangular slot of height $H=10 \mathrm{~mm}$ and width $W=135 \mathrm{~mm}$. The experiments confirmed the presence of the Undisturbed region of flow. Average fluid dynamics measurements in presence of a circular cylinder, impinged by the air jet, were carried out by Gori and Petracci (2003c) in the wind tunnel WT2, at $\operatorname{Re}=23,300$, with the conclusion that the presence of the impinged cylinder reduces the length 
of the Undisturbed Region of Flow. Shadowgraph visualizations and Hot Film Anemometer (HFA) measurements were carried out by Gori and Nino (2003) and Gori et al. (2007a) in a rectangular jet of air with the two wind tunnels WT1 and WT2. The experiments showed the dependence of the length $L_{C H}$ of the flow with Constant Height on the Reynolds number.

The main conclusion of these experiments is the increase of the lengths of the Undisturbed region of flow, $L_{U}$, and of the flow with Constant Height, $L_{C H}$, with the decrease of the Reynolds number. The original figure of Gori et al. (2007a) has been generalized in Gori et al. (2013) showing, besides the length of the line source, $L_{S}$, origin of the zone of established flow, the length of the Undisturbed Region of Flow, $L_{U}$, and the origin of the region of mixing fluid, $x_{0}$ and $y_{0}$. The value of $y_{0}$ is smaller than the half height of the slot, $H / 2$, because the experimental velocity profile on the slot exit is not flat and the interface of the region of mixing fluid is smaller than $H / 2$. On the other hand, the value of $x_{0}$ is smaller than $L_{U}$ for similar reasons. In other words, if the velocity profile would be exactly flat at the end of the Undisturbed Region of Flow, then $x_{0}=L_{U}$ and $y_{0}=H / 2$.

The experiments of Gori et al. (2013) confirmed, with average PIV visualizations and measurements, that the length of the average Undisturbed Region of Flow, $L_{U}$, decreases with the increase of the Reynolds number. The main characteristics of the average Undisturbed Region of Flow is that the average velocity maintains a profile almost equal to that on the exit, and can be identified by a constant height of the average PIV visualization, with length $L_{C H}$, or by an average constant turbulence on the centerline, with length $L_{C T}$.

The presence of the region of constant height has never been mentioned in the literature of turbulent free jet, even if some experimental visualizations, reported by van Dyke (1982) for axisymmetric free jets in turbulent flow, show its presence. Further
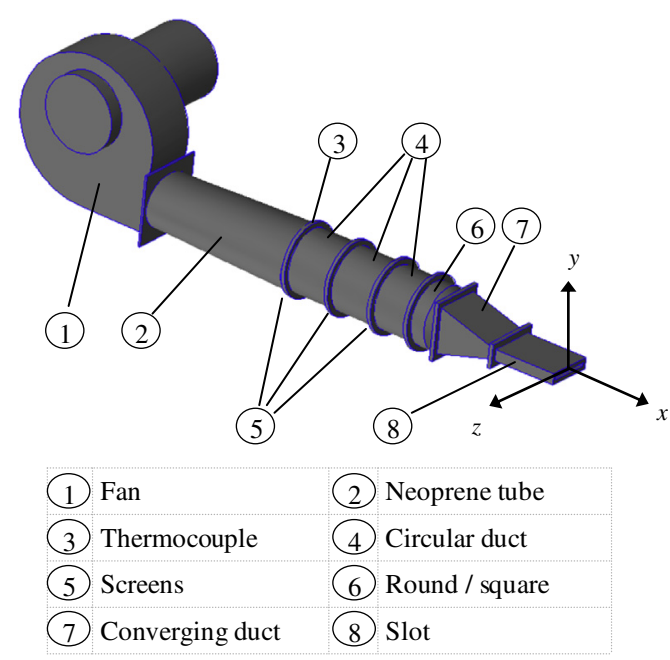

Fig. 1. Wind tunnel, WT1.

on, experiments on axisymmetric free helium jet, entering into air in laminar flow, and visualized with Schlieren and shadowgraph techniques, Goldstein (1996), show that the length of the region of constant height, in laminar flow, decreases from $L_{C H}=11.7-15.7 D$ at $\operatorname{Re}=80$, to $L_{C H}=2.3-2.7 D$ at $\operatorname{Re}=630$.

The modern technique of the Particle Image Velocimetry (PIV) allows the instant visualizations of rectangular jets, as reported by Gogineni and Shih (1997), who investigated free and wall jets, mostly in laminar flow, showing instant images without PIV velocity measurements. The instant visualizations confirmed that in laminar flow, i.e. from $\operatorname{Re}_{H}=330$ until $\operatorname{Re}_{H}=2200$, is present a region of constant height, with a length decreasing with the
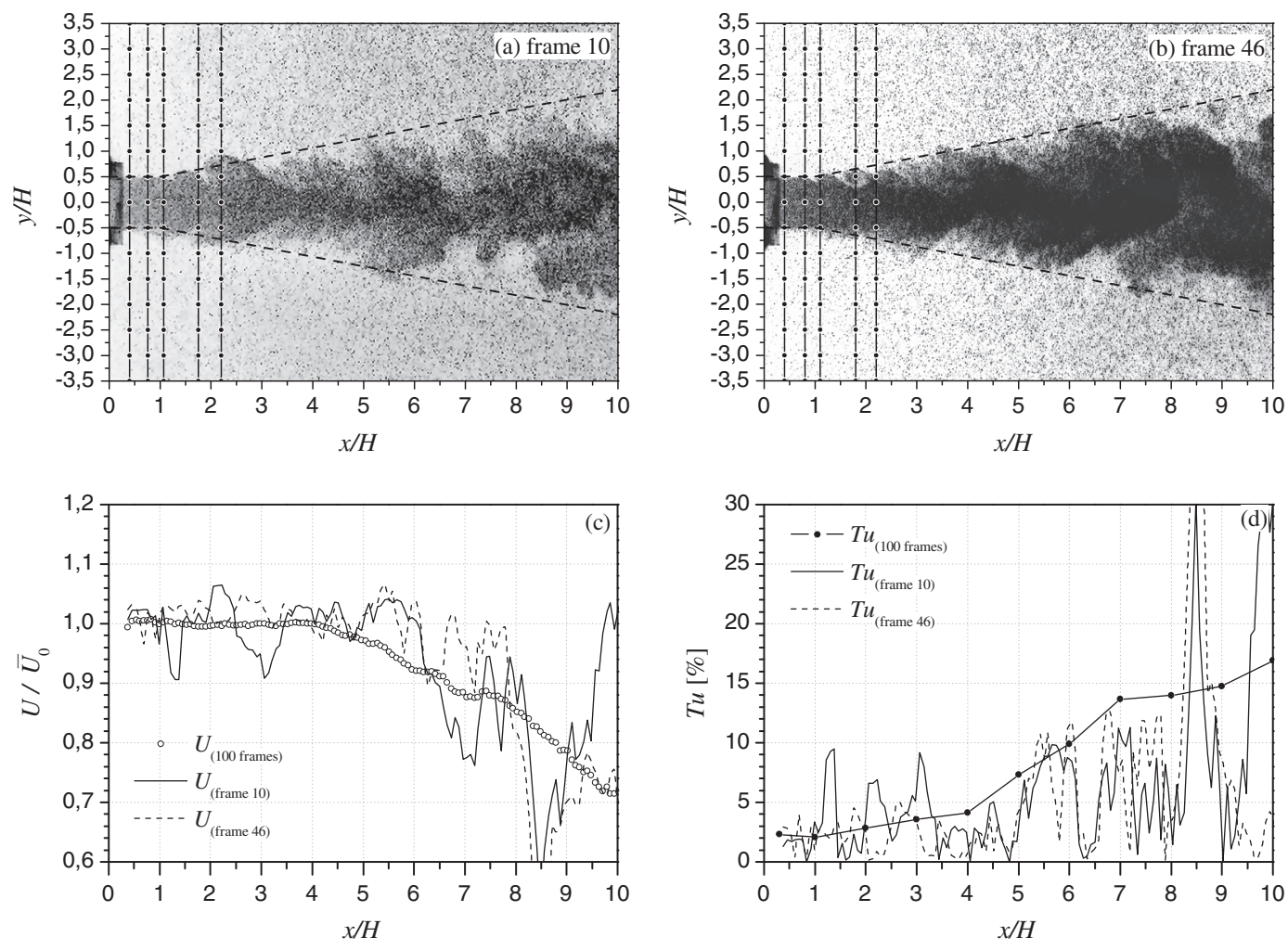

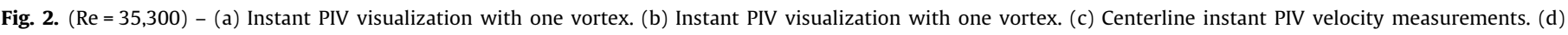
Centerline instant PIV turbulence intensity measurements. 
Table 1

Reynolds numbers of the instant PIV experiments and lengths of the flows ( $\mathrm{V}=$ visual inspection of PIV images, $\mathrm{M}=\mathrm{PIV}$ measurement of velocity or turbulence).

\begin{tabular}{|c|c|c|c|c|c|c|c|c|c|}
\hline $\mathrm{Re}$ & $\begin{array}{l}L_{\mathrm{CIH}} / H \\
\mathrm{~V}\end{array}$ & $\begin{array}{l}L_{C I V} / H, U / U o< \pm 4 \% \\
\mathrm{M}\end{array}$ & $\begin{array}{l}L_{C I T} / H, T u<4 \% \\
\mathrm{M}\end{array}$ & $\begin{array}{l}L_{U V P} / H \\
\mathrm{M}\end{array}$ & $L_{N D} / H=L_{1} / H$ & $\begin{array}{l}\left(L_{C I H}+L_{S V H}\right) / H \\
\mathrm{~V}\end{array}$ & $\begin{array}{l}\left(L_{C I T}+L_{S V T}\right) / H \\
\mathrm{M}\end{array}$ & $L_{2} / H$ & $\begin{array}{l}L_{3} / H \\
\mathrm{~V}\end{array}$ \\
\hline 35,300 & 0.9 & 1.1 & 1.1 & 1.1 & 1.05 & $1.8-1.6$ & $\begin{array}{l}T u<5 \% \\
1.8-1.3\end{array}$ & 1.62 & 4.5-Und \\
\hline 22,000 & 1.0 & 1.1 & 1.2 & 1.1 & 1.1 & $1.9-1.6$ & $\begin{array}{l}T u<5 \% \\
2.1-1.7\end{array}$ & 1.82 & Und-5.0 \\
\hline 11,200 & 2.5 & 2.7 & 2.7 & 2.5 & 2.6 & $3.2-2.8$ & $\begin{array}{l}T u<5 \% \\
3.3-2.8\end{array}$ & 3 & $\begin{array}{l}5.2-6.2 \\
(5.7)\end{array}$ \\
\hline 6800 & 2.9 & 3.3 & 3.2 & 3.0 & 3.1 & $4.2-3.4$ & $\begin{array}{l}T u<5 \% \\
4.3-3.3\end{array}$ & 3.85 & $\begin{array}{l}6.5-7.0 \\
(6.7)\end{array}$ \\
\hline 3400 & $3.1-2.9$ & 3.4 & 3.4 & 3.3 & 3.3 & $4.8-4.2$ & $\begin{array}{l}T u<9 \% \\
4.8-4.2\end{array}$ & 4.6 & $\begin{array}{l}7.0-7.5 \\
(7.25)\end{array}$ \\
\hline 2200 & 4.0 & 4.1 & 4.1 & 4.1 & 4.1 & $5.9-5.5$ & $\begin{array}{l}T u<8 \% \\
5.2\end{array}$ & 5.6 & $\begin{array}{l}8.0-9.0 \\
(8.5)\end{array}$ \\
\hline
\end{tabular}

increase of the Reynolds number, and is followed by the region of coherent vortices, and by the vortices breakdown. The only image of Gogineni and Shih (1997) in turbulent flow shows the disappearance of the coherent vortices and the vortices breakdown just after the jet exit. Instant images of a rectangular jet at the early stage of turbulent flow, $\operatorname{Re}_{H}=4240$, have been presented by Potho and Longmire (2001), showing a short region of constant height, followed by a region of coherent vortices and by the vortices breakdown. The PIV velocity measurements were only the average ones. The smoke flow visualization was employed by Hsiao et al. (2010) to study the flow structure of a sharp-edged and right-angle orifice plane jet in transient flow conditions at $\mathrm{Re}_{H}=10,300$. The images showed varicose and sinuous modes of the coherent structures, a short region of constant height before the coherent vortices flow, followed by the vortices breakdown. No instant flow measurements were shown.

The present paper presents instant Particle Image Velocimetry (PIV) visualizations and measurements in the flow evolution of rectangular free jets of air in the range of Reynolds numbers from $\operatorname{Re}=35,300$ to $\operatorname{Re}=2200$, with the aim of showing the dependence on the Reynolds number of the lengths of the flows identified by the paper. A correct description of the fluid dynamics evolution of a rectangular jet is extremely important in the numerical predictions, as shown by Gori and Petracci (2003d) and Gori et al. (2012a), in the heat transfer upon a single smooth cylinder, by Gori and Bossi (2000a) and (2003), Gori and Petracci (2003a), Gori et al. (2003b), Gori et al. (2012b), Gori and Petracci (2012, 2013) and (2014), upon a finned cylinder, by Gori et al. (2002b, 2003a, 2005, and 2011), upon two cylinders, by Gori and Bossi (2002), Gori et al. (2007b) and upon three cylinders, by Gori and Coppa (1998), Gori and Bossi (2000b).

\section{Experimental apparatus}

The wind tunnel employed in the present experiments, WT1, is shown in Fig. 1 and is the same of that of Gori et al. (2013).

The fan, with a power of $750 \mathrm{~W}$, can move a maximum mass flow rate of $0.45 \mathrm{~kg} / \mathrm{s}$. The inlet duct of the fan has a circular cross section of diameter $160 \mathrm{~mm}$ and the exit duct has a square section of side $135 \mathrm{~mm}$. The fan lies on a different base than the rest of the wind tunnel, in order to reduce vibrations, and is followed by a settling chamber, made of a non-metallic (plastic) tube to reduce vibrations, where the cross section is changed from rectangular (at the outlet of the fan) to circular, with a diameter of $160 \mathrm{~mm}$. After the settling chamber, honeycombs are located before the three metallic grids. Honeycombs consist of several small-size ducts of circular section that fill the entire cross sectional area of the duct minimizing the transversal flows, while the grid screens reduce the large-scale turbulence generated by

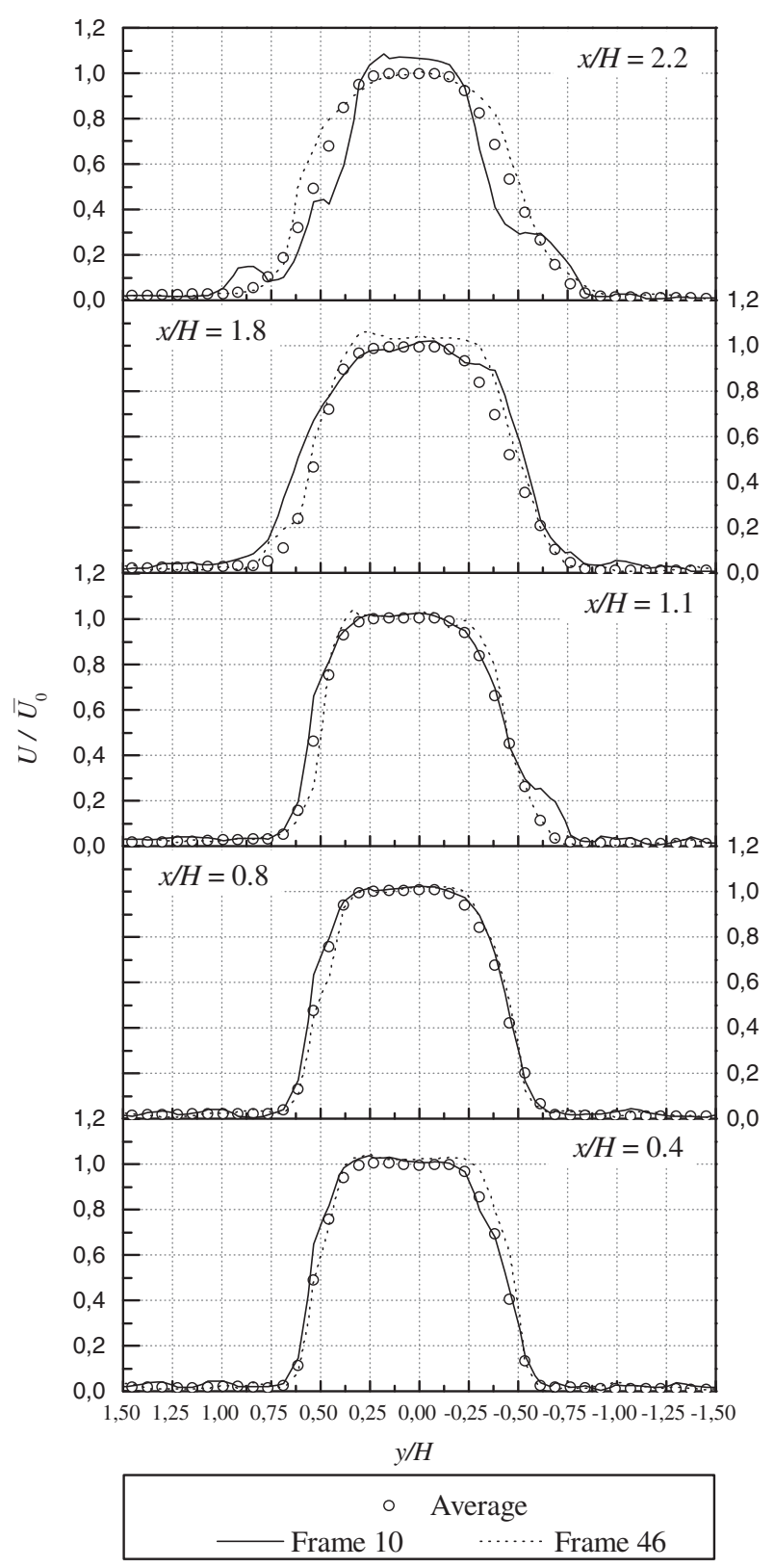

Fig. 3. Instant PIV velocity measurements for $\operatorname{Re}=35,300$.

the fan. After the third grid, the cross section of the wind tunnel changes into a square one with the side of $106 \mathrm{~mm}$. A converging duct converts the square cross section to a rectangular one, with 

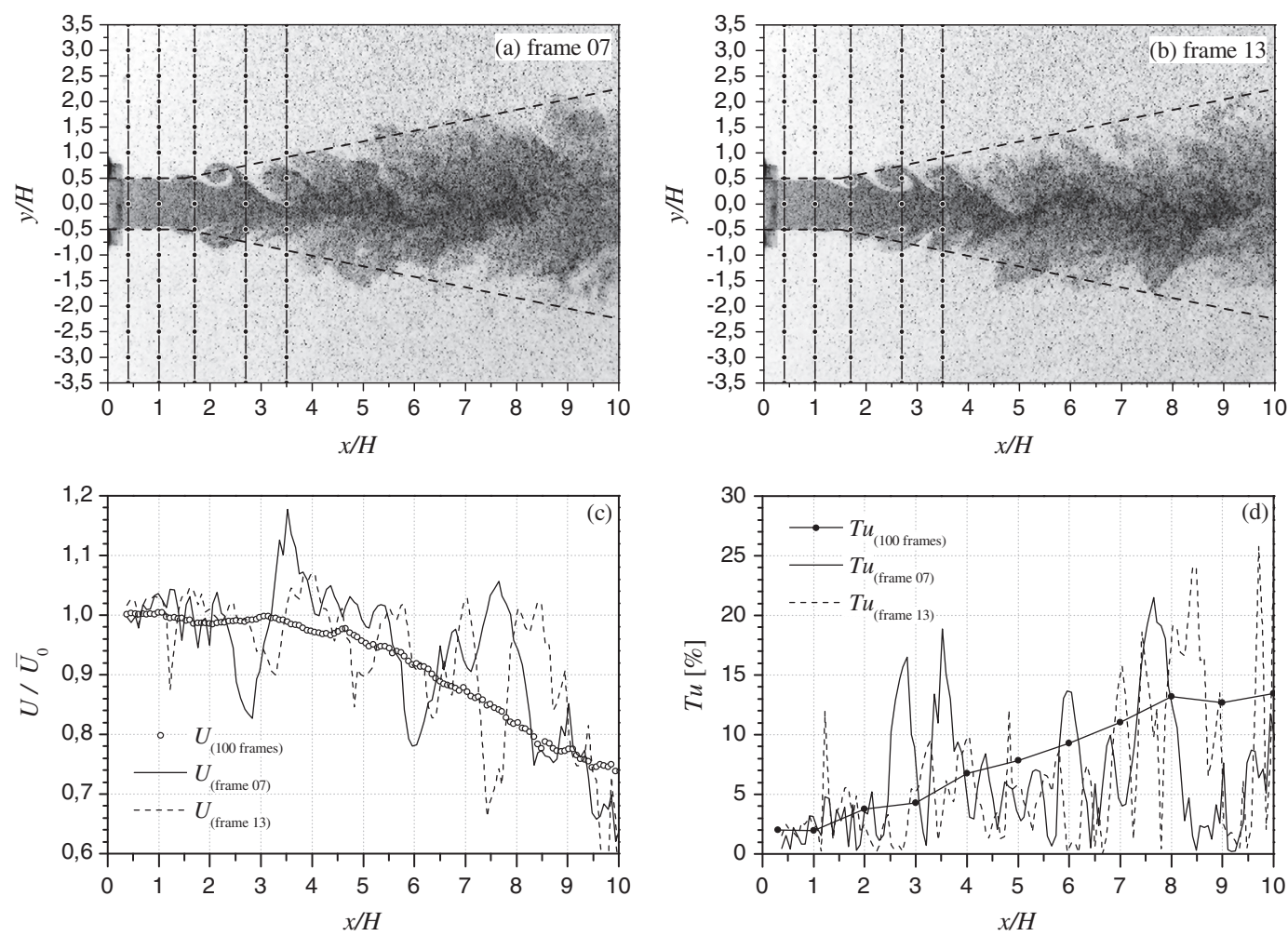

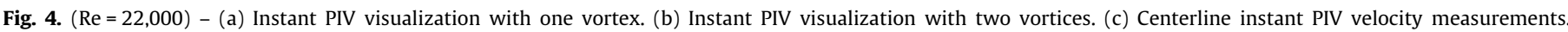
(d) Centerline instant PIV turbulence intensity measurements.

sides $106 \mathrm{~mm}$ by $17 \mathrm{~mm}$. A rectangular slot follows the converging nozzle.

\subsection{PIV setup}

The present investigation employs a commercially available PIV system, developed by ILA GmbH, where an ILA-TEC30 jet atomizer, with multiple Laskin nozzles in $x$-configuration (two layers), generates an oil-based aerosol. A cyclone separator, placed downstream the seeding generator, allows a mean particle diameter of about $10^{-6} \mathrm{~m}$, while the oil concentration is regulated by the compressed air pressure and the number of Laskin nozzles.

A Quantel Q-switched Nd:YAG laser provides pulsed illuminations with a wavelength of $532 \times 10^{-9} \mathrm{~m}$. The duration of each pulse is $3-5 \times 10^{-9} \mathrm{~s}$, and the maximum output energy is $0.120 \mathrm{~J}$. The light-sheet thickness in the test section is $1 \mathrm{~mm}$. The digital camera is a PCO Sensicam QE, containing a CCD chip with $1376 \times 1040$ pixels, a dynamic range A/D of 12 bit, a frame rate equal to $10.0 \mathrm{fps}$ with $16 \mathrm{MHz}$ of pixel scan rate. The camera and a Nikon AF Micro 50f/1.4D lens with an optical filter record the particle images. The size of the interrogation window is $200 \mathrm{~mm}$ by $140 \mathrm{~mm}$, respectively in $x$ and $y$ direction, giving rise to the spatial resolution of $6.55 \mathrm{pixel} / \mathrm{mm}$. The time delay between two successive pulses varies from 20 to $500 \times 10^{-6} \mathrm{~s}$, depending on the tested flow conditions. An ensemble of 100 instantaneous velocity samples is generated with an acquisition rate of $5 \mathrm{~Hz}$.

The software 'vidPIV' carries out the image processing applying a cross-correlation algorithm between consecutive images. The PIV processing uses an interrogation area size of $16 \times 16$ pixels, with a $50 \%$ overlap. A window velocity filter and a local median filter are employed to remove outliers (local velocity vectors whose magnitude is far from the neighbor set of data) in order to obtain a clear velocity field, after the correlation of the images. Finally, interpolation of the neighboring vectors replaces all the vectors previously removed as outliers. The resulting vectors are averaged over the 100 realizations.

Uncertainty analysis is carried out by using the engineering method proposed by Moffat (1988). In case of multiple samples, each measurement of the physical quantity $X_{i}$ is represented by the mean sample value and the uncertainty is calculated using the bias $\left(B_{X i}\right)$ and the average precision index $\left(S_{X i}\right)$, which describes respectively the fixed error and the sample standard deviation. Both are calculated on the basis of the sensitivity to the specific error source. The PIV measurement detects the flow speed by means of the displacement of particle images $\Delta \chi$, the time interval of successive images $\Delta t$ (pulse distance) and the magnification factor $M$. The physical amount of flow speed is calculated according to the relation

$U=M \frac{\Delta \chi}{\Delta t}$

The magnification factor needs to be identified through the calibration and is defined as

$\left.M=\frac{d \cos \vartheta}{D^{\prime}} \cong \frac{d}{D^{\prime}} \quad 1-\frac{\vartheta^{2}}{2}\right)$

where $\vartheta$ is the angle between the light sheet and the calibration board, $d$ the reference distance in the calibration board and $D^{\prime}$ represents the same distance on the image plane.

Taking into account Eq. (2), the main error sources due to the evaluation of $M$ are related to the measurement of the distance $d$ on the calibrated board, with an uncertainty of $0.5 \mathrm{~mm}$, and to the measurement of the distance $D^{\prime}$ on the image plane, with an uncertainty of 0.5 pixels. The possible small angle between the 


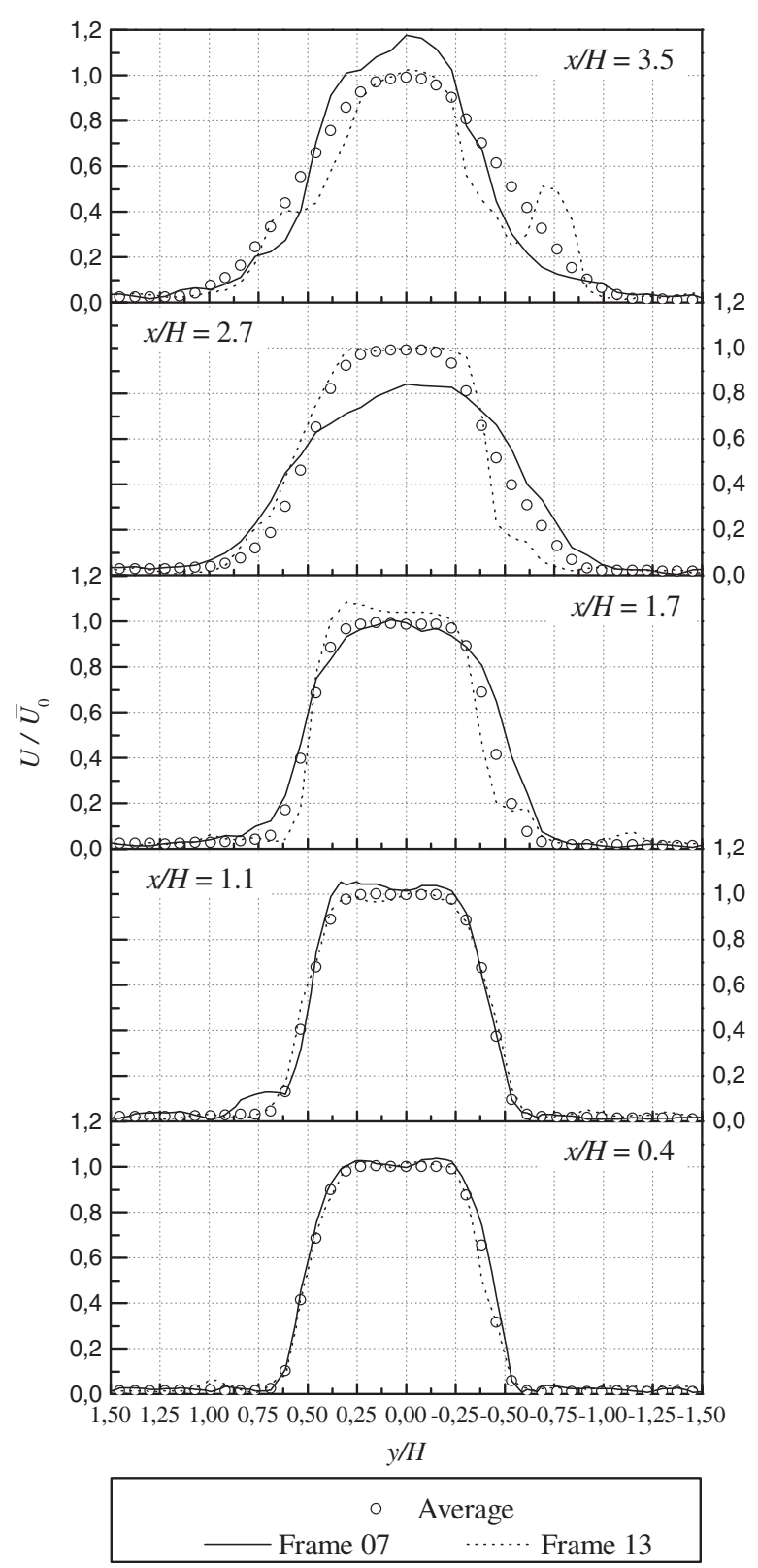

Fig. 5. Instant PIV velocity measurements for $\mathrm{Re}=22,000$.

light sheet and the calibration board is neglected. The evaluation of the displacement of the particle image $(\Delta \chi)$ is affected by many sources of error related to the visualization, the image detection and especially the data processing. The error in the data processing is due to the accuracy in the peak detection during the cross-correlation analysis and is function of the size of the interrogation window, number of particles, local velocity gradients inside the interrogation window, non-matching particles, and noise. Therefore, the value of 0.1 pixels can be considered reasonable under real measurements. This condition is more easily satisfied if the time between pulses is chosen to ensure that the maximum displacement does not exceed a quarter of the side of the interrogation area, as recommended by Keane and Adrian (1990). The random uncertainty in the timing is of the order of $1 \mathrm{~ns}$, according to the synchronizer. For the minimum pulse separation used in the present work $(20 \mu \mathrm{s})$, the uncertainty due to $\Delta t$ is then negligible compared to the displacement uncertainty.
All the uncertainties are evaluated under three conditions:

1. Error distribution is Gaussian according to the central limit theorem.

2. $X_{i}$ variable contribution to the global uncertainty is reciprocally independent.

3. Measurement accuracy is expressed with a confidence of $95 \%$.

All the samples of measurement are multiple, with 100 acquisitions.

For each physical quantity, $X_{i}$, the uncertainty is expressed by the following equation:

$\delta X_{i-0.95}=\left[B_{X_{i}}^{2}+\left(t \cdot S_{\bar{X}_{i}}\right)^{2}\right]^{\frac{1}{2}}$

The value of the multiplier $t$ (Student's test) is set to 2 according to the confidence interval (95\%) and the sample degrees of freedom (more than 30). Moreover, the definition used is

$S_{\bar{X}_{i}}=\frac{S_{X_{i}}}{\sqrt{N}}$

The measurement error theory suggests that the result $R$ of an experiment can be determined by a series of $N$ measurements, a single quantity $X_{i}$, and an interpolating criterion, thus

$R=R\left(X_{1}, X_{2}, \ldots, X_{i}, \ldots, X_{N}\right)$

The $R$ result for measurement bias and precision index are described by

$B_{R}=\left[\sum_{i=1}^{N}\left(\frac{\partial R}{\partial X_{i}} \cdot B_{X_{i}}\right)^{2}\right]^{\frac{1}{2}}$
$S_{R}=\left[\sum_{i=1}^{N}\left(\frac{\partial R}{\partial X_{i}} \cdot S_{X_{i}}\right)^{2}\right]^{\frac{1}{2}}$

The global uncertainty can be expressed by:

$\delta R_{0.95}=\left[B_{R}^{2}+\left(t \cdot S_{R}\right)^{2}\right]^{\frac{1}{2}}$

Finally, in the operating conditions of the experiments (flow regime and distance from the slot exit) the uncertainty on the average velocity is smaller than $4 \%$.

\subsection{Jet flow}

The volume flow rate is measured by a pressure transducer (mod. MD OD8612 L2 "Orione di Bistulf") connected to two static pressure probes. One probe is positioned on the wall of the settling chamber (larger section), i.e. before the converging duct, while the other one is on the lateral wall (smaller section), i.e. beyond the converging duct. The static pressure difference, proportional to the flow rate, is corrected taking into account the pressure losses due to friction in the converging duct. The volume flow rate, $\dot{V}$, obtained by the pressure measurements, is checked by the integration of the velocity profile on the slot exit $(x / H=0.3)$.

The average flow velocity $U_{a v}$ is obtained from the volume flow rate $\dot{V}$

$U_{a v}=\frac{\dot{V}}{W \cdot H}$

The Reynolds number is defined on the hydraulic diameter, $D$

$\operatorname{Re}=\frac{U_{a v} \cdot D}{v}$ 

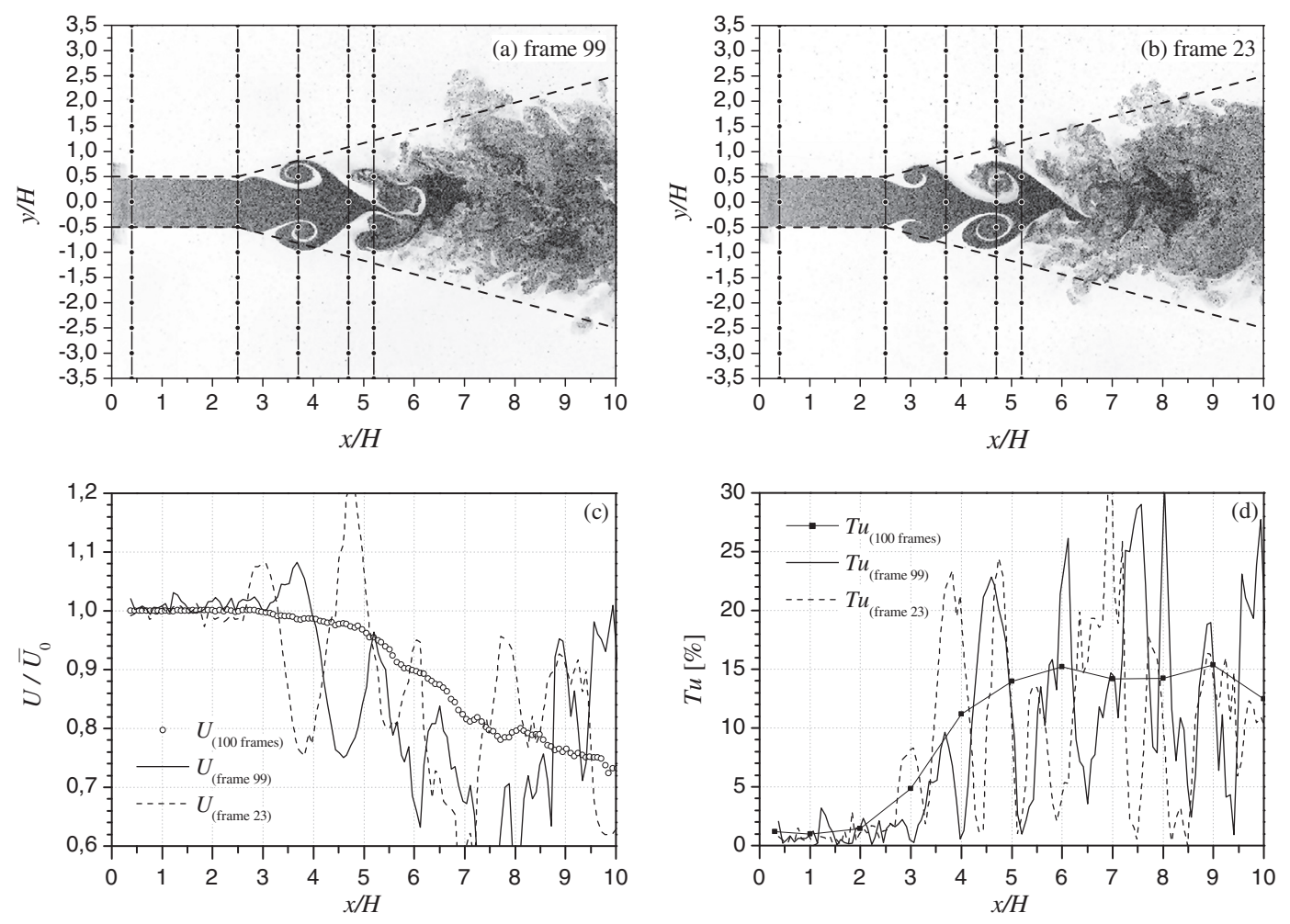

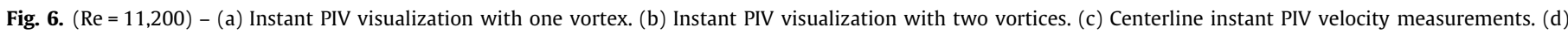
Centerline instant PIV turbulence intensity measurements.

Turbulence intensity is the absolute turbulence and is defined on the basis of the time average velocity on centerline of the slot exit. Turbulence is calculated for 100 couples of frames of the PIV acquisition and a single couple ( $j$ th frame) as follows:

$$
\begin{aligned}
& T u_{(100 \text { frame })}=\frac{R M S\left(U^{\prime}\right)}{\bar{U}_{0}}[\%]=\frac{1}{\bar{U}_{0}} \sqrt{\frac{1}{N-1} \sum_{N=1}^{100}(U-\bar{U})^{2}}[\%] \\
& T u_{(\text {frame “j") }}=\frac{\left|U_{j}-\bar{U}_{j}\right|}{\bar{U}_{0}}[\%]=\frac{\left|U_{j}^{\prime}\right|}{\bar{U}_{0}}[\%]
\end{aligned}
$$

\section{Experimental results}

\subsection{Dependence of flow evolution on Reynolds number in turbulent regime}

The instant PIV visualizations and measurements in turbulent flow are presented and discussed for Reynolds numbers equal to $\operatorname{Re}=35,300, \operatorname{Re}=22,000, \operatorname{Re}=11,200, \operatorname{Re}=6800$ and $\operatorname{Re}=3400$.

Two instant PIV visualizations with one and two vortices are examined for each Reynolds number, except for $\operatorname{Re}=35,300$ where only images with one vortex have been detected. The measurements of the instant PIV dimensionless velocity, $U / \bar{U}_{0}$, on the centerline, and turbulence intensity, $T u$, are reported versus the dimensionless distance from the exit, $x / H$, along with the average measurements of Gori et al. (2013), indicated as 100 frames. The profiles of the instant PIV dimensionless velocity measurements, $U / \bar{U}_{0}$, versus the dimensionless transverse coordinate, $y / H$, are shown at five distances from the exit for each Reynolds number and compared to the average ones. The first profile is very close to the exit one, while the others are inside the potential core.

\subsection{1. $R e=35,300$}

3.1.1.1. Visual inspection. Two instant flow visualizations with one vortex are reported in Fig. 2a, frame 10, and 2b, frame 46, at the highest Reynolds number investigated, $R e=35,300$. Each figure shows the presence of the Flow with Constant Instant Height, $\mathrm{F}_{\mathrm{CIH}}$, the Flow with Small Variation of the Height, $\mathrm{F}_{\mathrm{SVH}}$, the Flow with Coherent Vortices, $\mathrm{F}_{\mathrm{CV}}$ and the vortices breakdown. The dashed lines represent the average flow of Gori et al. (2013), which envelopes correctly the instant visualizations. Each figure indicates the five marks where the instant velocity profiles are measured.

Fig. 2a presents the instant visualization of frame 10 with the flow $\mathrm{F}_{\mathrm{CIH}}$ of length $L_{\mathrm{CIH}} / H=0.9$, and the flow $\mathrm{F}_{\mathrm{SVH}}$ up to $x / H=1.8$, then $\left(L_{C H}+L_{S V H}\right) / H=1.8$. The end of the flow $\mathrm{F}_{\mathrm{CV}}$ is in the range $x / H=4-5$, i.e. $\left(L_{C I H}+L_{S V H}+L_{C V}\right) / H=L_{3} / H=4.5$. Fig. 2 b reports the visualization of frame 46 with the flow $\mathrm{F}_{\mathrm{CIH}}$ of length $L_{\mathrm{CIH}} / H=0.9$ and the flow $\mathrm{F}_{\mathrm{SVH}}$ up to $x / H=1.6$, i.e. $\left(L_{C I H}+L_{S V H}\right) / H=1.6$. The end of the flow $\mathrm{F}_{\mathrm{CV}}$ is not identifiable in Fig. 2b. The average visualization of Gori et al. (2013) envelopes correctly the instant flow evolutions of Fig. 2a and b.

The lengths resulting from the visual inspection, $L_{C I H} / H$, $\left(L_{C I H}+L_{S V H}\right) / H$ and $\left(L_{C I H}+L_{S V H}+L_{C V}\right) / H=L_{3} / H$, are reported in Table 1 . The length of the flow $\mathrm{F}_{\mathrm{CIH}}, L_{\mathrm{CIH}} / H=0.9$, is in agreement with the average one of Gori et al. (2013), $L_{C H} / H=1.0$.

3.1.1.2. PIV measurements. Fig. 2c presents the measurements of the centerline instant dimensionless velocity, $U / \bar{U}_{0}$, versus the distance $x / H$ from the jet exit, compared to the average ones of Gori et al. (2013), as 100 frames. The instant velocities of both frames 10 and 46 are variable within $\pm 4 \%$ up to $x / H=1.1$, defining the flow with Constant Instant Velocity on the centerline $\mathrm{F}_{\mathrm{CIV}}$, of length $L_{C I V} / H=1$.1. After $L_{C I V}$, velocity has greater oscillations, with frame 10 which presents two minimums in the two section enlargements, $x / H=1.3$ and $x / H=3.0$, and a maximum in the section contraction, $x / H=2.2$. Frame 46 presents a velocity maximum at 


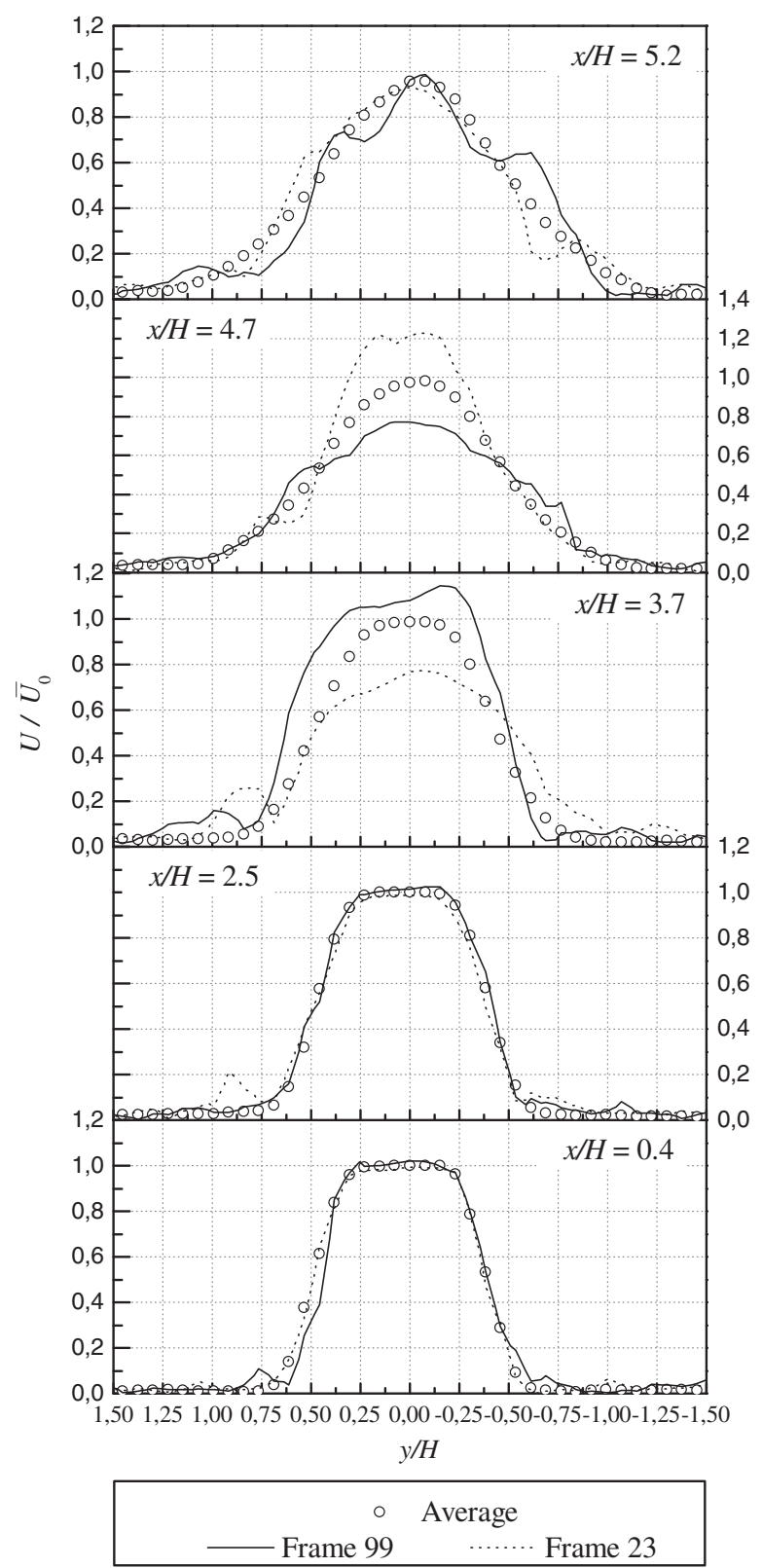

Fig. 7. Instant PIV velocity measurements for $\mathrm{Re}=11,200$.

$x / H=1.8$, where is present a small height contraction. These behaviors agree with the mass conservation or Leonardo principle.

Fig. $2 \mathrm{~d}$ presents the instant turbulence intensity measurements, $\mathrm{Tu}$, on the centerline, compared to the average ones of Gori et al. (2013), as 100 frames. Turbulent intensity of the two frames remains below $4.0 \%$ up to $x / H=1.1$, giving a length $L_{C I T} / H=1.1$ of the Flow with Constant Instant Turbulence $\mathrm{F}_{\mathrm{CIT}}$. After $L_{\mathrm{CIT}}$, turbulence intensity of frame 10 increases, but remaining below $5 \%$ up to $x / H=1.3$, i.e. $\left(L_{C I T}+L_{S V T}\right) / H=1.3$, and having a spike of $9 \%$ at $x / H=1.4$, i.e. in correspondence with the first small contraction of the height. A second spike of $7 \%$ is present before the first vortex, at $x / H=2.1$, followed by another one of $9 \%$, at $x / H=3.1$, where is present the vortex. After $L_{C I T}$, turbulence intensity of frame 46 remains below $5 \%$ up to $x / H=1.8$, i.e. $\left(L_{C I T}+L_{S V T}\right) / H=1.8$.

The lengths of the flows $\mathrm{F}_{\mathrm{CIV}}, L_{\mathrm{CIV}} / H=1.1$, and $\mathrm{F}_{\mathrm{CIT}}, L_{C I T} / H=1.1$, are reported in Table 1 showing their agreement with the length of the flow $\mathrm{F}_{\mathrm{CIH}}, L_{\mathrm{CIH}} / \mathrm{H}=0.9$, and the average length of the flow with constant turbulence, $\mathrm{F}_{\mathrm{CT}}$, of Gori et al. (2013), $L_{C T} / H=1.0$. The length $\left(L_{C I T}+L_{S V T}\right) / H=1.3$ of frame 10 is slightly smaller than the length $\left(L_{C H}+L_{S V H}\right) / H=1.6$ of the same frame, while the length $\left(L_{C I T}+L_{S V T}\right) / H=1.8$ of frame 46 is equal to $\left(L_{C I H}+L_{S V H}\right) / H=1.8$ of the same frame. The length $\left(L_{C I T}+L_{S V T}\right) / H$ is shorter for frame 13 due to the presence of the second vortex.

Fig. 3 presents the dimensionless instant velocity measurements, $U / \bar{U}_{0}$, versus the transverse dimensionless distance, $y / H$, at five distances from the slot exit, corresponding to the marks of Fig. 2. The first distance, $x / H=0.4$, is the closest to the exit and its profile is assumed as the exit one. The instant measurements of frames 10 and 46 are in agreement with the average one at $x / H=0.4$. The same happens at the second distance, $x / H=0.8$, and at the third one, $x / H=1.1$. The two instant measurements at $x / H=1.1$ are practically unchanged in comparison to the exit values, at $x / H=0.4$, marking the end of the flow with Unchanged Velocity Profile, $F_{U V P}$, of length $L_{U V P} / H=1.1$, in agreement with the average value of the Undisturbed Region of Flow of Gori et al. (2013), $L_{U} / H=1.0$. At the fourth distance, $x / H=1.8$, some differences between the instant velocity profiles of frames 10 and 46 are observable, both on the centerline and the sides. The velocity profile of frame 46 , presented as dashed line, has slightly greater values than the average ones in its inner part because the jet has a height contraction, due to the mass conservation principle. At the fifth distance, $x / H=2.2$, the velocity profile of frame 10 is greater around the centerline and smaller on the jet sides because of the height contraction, while the height and the velocity are almost unchanged for frame 46.

The length of the flow with Unchanged Velocity Profile, $F_{U V P}$, $L_{U V P} / H=1.1$, is reported in Table 1 , and is in agreement with the lengths $L_{C I H} / H, L_{C I V} / H$ and $L_{C I T} / H$.

\subsection{2. $R e=22,000$}

3.1.2.1. Visual inspection. Fig. 4a presents the instant visualization of frame 07 with one vortex, and Fig. $4 \mathrm{~b}$ that of frame 13 with two vortices, while the instant centerline velocity and turbulence measurements are in Fig. 4c and d. Fig. $4 \mathrm{a}$ and b shows also the average flow of Gori et al. (2013), which envelopes correctly the instant visualizations. The five marks of Fig. $4 a$ and $b$ indicate the positions of the instant velocity profile measurements.

Frame 07 of Fig. 4a shows that the flow $\mathrm{F}_{\mathrm{CIH}}$ is long $L_{\mathrm{CIH}} / H=1.0$, the flow $\mathrm{F}_{\mathrm{SVH}}$ extends up to $x / H=1.9$, then $\left(L_{\mathrm{ClH}}+L_{\mathrm{SVH}}\right) / H=1.9$, and is followed by one vortex and the vortices breakdown. The end of the potential core is not easily identifiable in Fig. 4a. Frame 13 of Fig. $4 \mathrm{~b}$ shows a similar length of the flow $\mathrm{F}_{\mathrm{CIH}}, L_{\mathrm{CIH}} / H=1.0$, a shorter length of the flow $F_{S V H}$, up to $x / H=1.6$, then $\left(L_{C I H}+L_{S V H}\right) / H=1.6$, which is followed by a first small vortex, by a greater second one and by the vortices breakdown. The length $\left(L_{C I H}+L_{S V H}\right) / H$ is shorter in frame 13 because two vortices are present. The end of the coherent vortices in frame 13 can be identified at $L_{3} / H=5.0$.

The results of the visual inspection, $L_{C I H} / H,\left(L_{C I H}+L_{S V H}\right) / H$, and $L_{3} / H$, are reported in Table 1 , with the first value relative to the frame with one vortex and the second one to the frame with two vortices. The length of the flow $\mathrm{F}_{\mathrm{ClH}}, L_{\mathrm{ClH}} / H=1.0$, is within the average range of Gori et al. (2013), $L_{C H} / H=1-2$.

3.1.2.2. PIV measurements. Fig. 4c presents the instant dimensionless centerline velocity measurements, $U / \bar{U}_{0}$, along with the average one, indicated as 100 frames, versus the distance $x / H$ from the jet exit. The dimensionless centerline velocities, $U / \bar{U}_{0}$, of the two frames are variable within $\pm 4 \%$ up to $x / H=1.1$, identifying the length of the flow $\mathrm{F}_{\mathrm{CIV}}, L_{\mathrm{CIV}} / H=1.1$. After $L_{C I V}$, frame 07 shows small oscillations, corresponding to little contractions and enlargements of the height. In correspondence of the enlargement of the height, at $x / H=2.8$, Fig. 4 a shows a velocity decrease of $18 \%$, which allows the pressure to increase, according to the Bernoulli equation, and to expel the fluid from the jet core to form the vortex. This vortex of frame 07 ends at $x / H=3.5$, where a velocity increase of about 

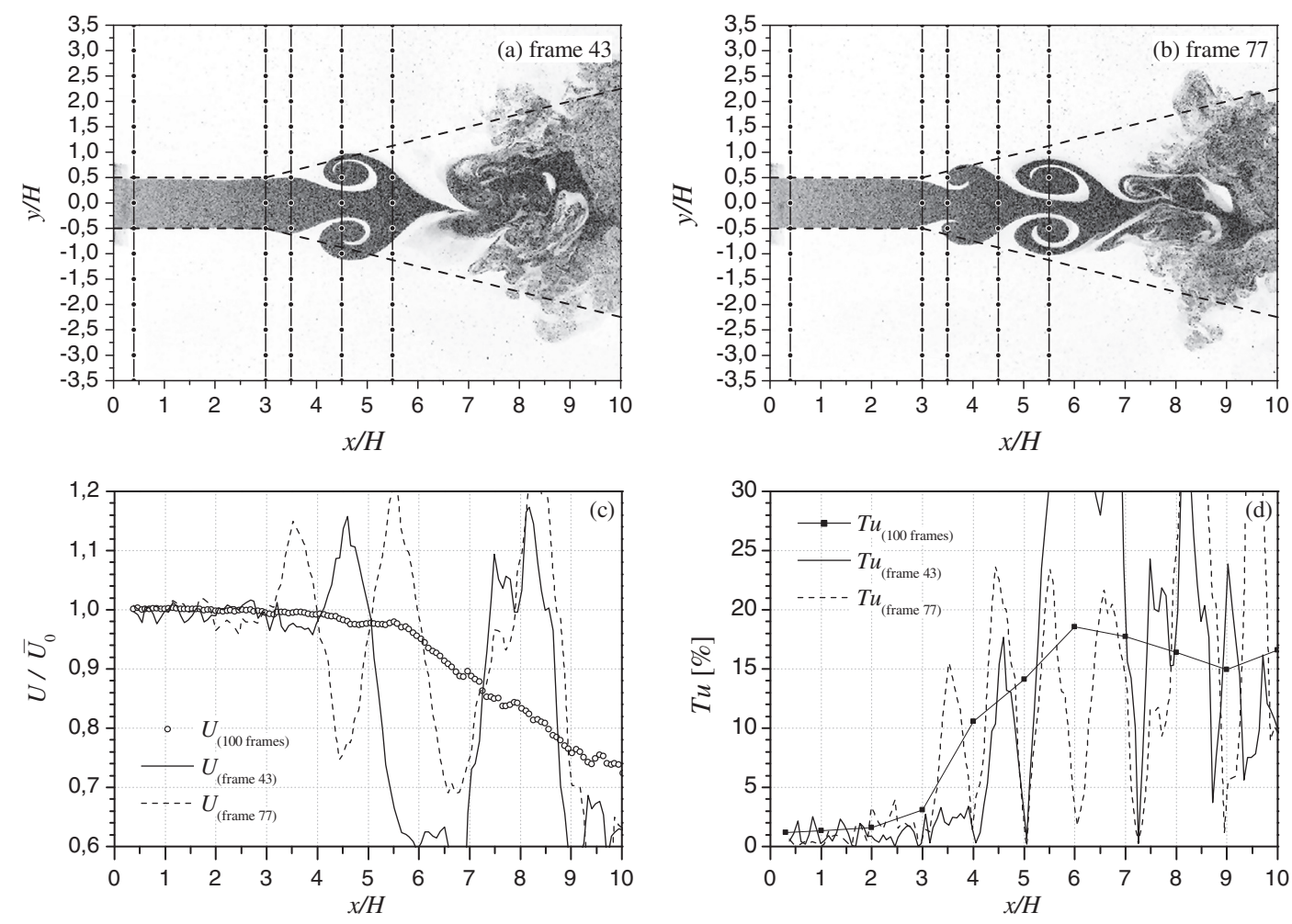

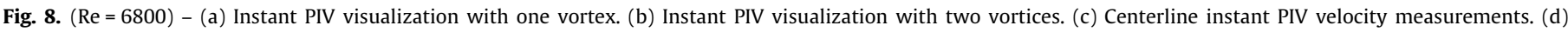
Centerline instant PIV turbulence intensity measurements.

$20 \%$ is present. Frame 13 shows two minimums, at $x / H=1.2$ and $x /$ $H=3.2$, corresponding to the enlargements of the height. The minimum of velocity at $x / H=3.2$ produces a pressure increase which, according to the Bernoulli equation, expels the fluid from the jet core to form the second coherent vortex.

Fig. $4 \mathrm{~d}$ presents the instant turbulence intensity measurements on the centerline. Turbulence intensity variations of both frames are smaller than $4.0 \%$ up to $x / H=1.2$, giving the length of the flow $\mathrm{F}_{\mathrm{CIV}}, L_{C I V} / H=1.2$. After $L_{C I V}$, turbulence intensity of frame 07 increases but remaining below $5 \%$ up to $x / H=2.1$, which allows to define the flow with Small Variations of the instant Turbulence, $\mathrm{F}_{\mathrm{SVT}}$, of length $L_{S V T}$, such that $\left(L_{C I T}+L_{S V T}\right) / H=2.1$. Frame 07 presents a spike of turbulence at $x / H=2.8$, in correspondence with the minimum of the velocity and the formation of the vortex. After $L_{C I V}$ frame 13 presents an isolate spike of the turbulence intensity at $x / H=1.2$, but, apart from that, remains smaller than $5 \%$ up $x / H=1.6$, giving a length $\left(L_{C I T}+L_{S V T}\right) / H=1.6$. Frame 13 shows another spike at $x / H=3.2$, in correspondence with the second vortex.

The lengths of the flows $\mathrm{F}_{\mathrm{CIV}}, L_{\mathrm{CIV}} / H=1.1$, and $\mathrm{F}_{\mathrm{CIT}}, L_{\mathrm{CIT}} / H=1.2$, are reported in Table 1 showing they are in agreement with the flow $\mathrm{F}_{\mathrm{ClH}}, L_{\mathrm{CIH}} / \mathrm{H}=1.0$, and are within the average length of Gori et al. (2013), $L_{C T} / H=1-2$. The length $\left(L_{C I T}+L_{S V T}\right) / H=2.1$ of frame 07 is comparable to the length $\left(L_{C I H}+L_{S V H}\right) / H=1.9$ of the same frame, while the length $\left(L_{C I T}+L_{S V T}\right) / H=1.7$ of frame 13 is comparable to $\left(L_{C I H}+L_{S V H}\right) / H=1.6$ of the same frame. The length $\left(L_{C I T}+L_{S V T}\right) / H$ is shorter for frame 13 , due to the presence of the second vortex.

Fig. 5 presents the instant dimensionless velocity measurements, $U / \bar{U}_{0}$, versus the transverse dimensionless distance, $y / H$, at five downstream distances from the slot exit, corresponding to the marks of Fig. $4 \mathrm{a}$ and $\mathrm{b}$. At the first distance, $x / H=0.4$, the closest to the exit, the instant measurements of frames 07 and 13 are both in agreement with the average one. The two instant measurements at $x / H=1.1$ are practically unchanged within the jet section, compared to the values at $x / H=0.4$, marking the end of the flow $\mathrm{F}_{\mathrm{UVP}}$, i.e. $L_{U V P} / H=1.1$. At the third distance, $x / H=1.7$, the velocity of frame 07 near the centerline is unchanged because the height is equal to the exit one, while the velocity of frame 13 is greater because of the height contraction. At the fourth distance, $x / H=2.7$, the velocity of frame 13 is still unchanged while the velocity of frame 07 near the centerline is smaller because of the height enlargement. At the fifth distance, $x / H=3.5$, it happens the opposite.

The length of the flow with Unchanged Velocity Profile, $\mathrm{F}_{\mathrm{UVP}}$, $L_{U V P} / H=1.1$, is reported in Table 1 , and is in agreement with the lengths $L_{C I H} / H=1.0, L_{C I V} / H=1.1, L_{C I T} / H=1.2$, and in the lower limit of the average range of Gori et al. (2013), $L_{U} / H=1-2$.

\subsection{3. $R e=11,200$}

3.1.3.1. Visual inspection. The two instant visualizations at $\operatorname{Re}=11,200$ are reported in Fig. 6a for frame 99 with one vortex, and in Fig. $6 \mathrm{~b}$ for frame 23 with two vortices. The centerline instant velocity and turbulence measurements are in Fig. $6 \mathrm{c}$ and d. Fig. 6a and $\mathrm{b}$ show also, as dashed line, the average flow which envelopes correctly the instant visualizations. In Fig. $6 a$ and $b$ the five marks indicate the positions of the instant velocity profile measurements.

Frame 99 shows the flow $\mathrm{F}_{\mathrm{ClH}}$ of length $L_{\mathrm{CIH}} / H=2.5$, the flow $\mathrm{F}_{\mathrm{SVH}}$ which extends up to $x / H=3.2$, i.e. $\left(L_{C I H}+L_{S V H}\right) / H=3.2$, followed by a large vortex and by the vortices breakdown. The end of the vortex is at $x / H=5.2$, then the total length is $L_{3} / H=5.2$. Frame 23 shows the flow $\mathrm{F}_{\mathrm{ClH}}$ of length $L_{\mathrm{CIH}} / \mathrm{H}=2.5$, followed by the flow $\mathrm{F}_{\mathrm{SVH}}$ up to $x / H=2.8$, i.e. $\left(L_{\mathrm{CIH}}+L_{S V H}\right) / H=2.8$. The flow $\mathrm{F}_{\mathrm{SVH}}$ is longer for frame 99 because only one vortex is present. The end of the second vortex at $x / H=6.2$ marks the total length for frame 23, $L_{3} / H=6.2$.

The results of the visual inspection, $L_{C I H} / H,\left(L_{C I H}+L_{S V H}\right) / H$, and $L_{3} / H$, are reported in Table 1 , with the first value relative to the frame with one vortex and the second one to the frame with two vortices. The length of the flow $\mathrm{F}_{\mathrm{ClH}}, L_{\mathrm{CIH}} / H=2.5$, is in agreement with the average values of Gori et al. (2013), $L_{C H} / H=2-3$, as well 


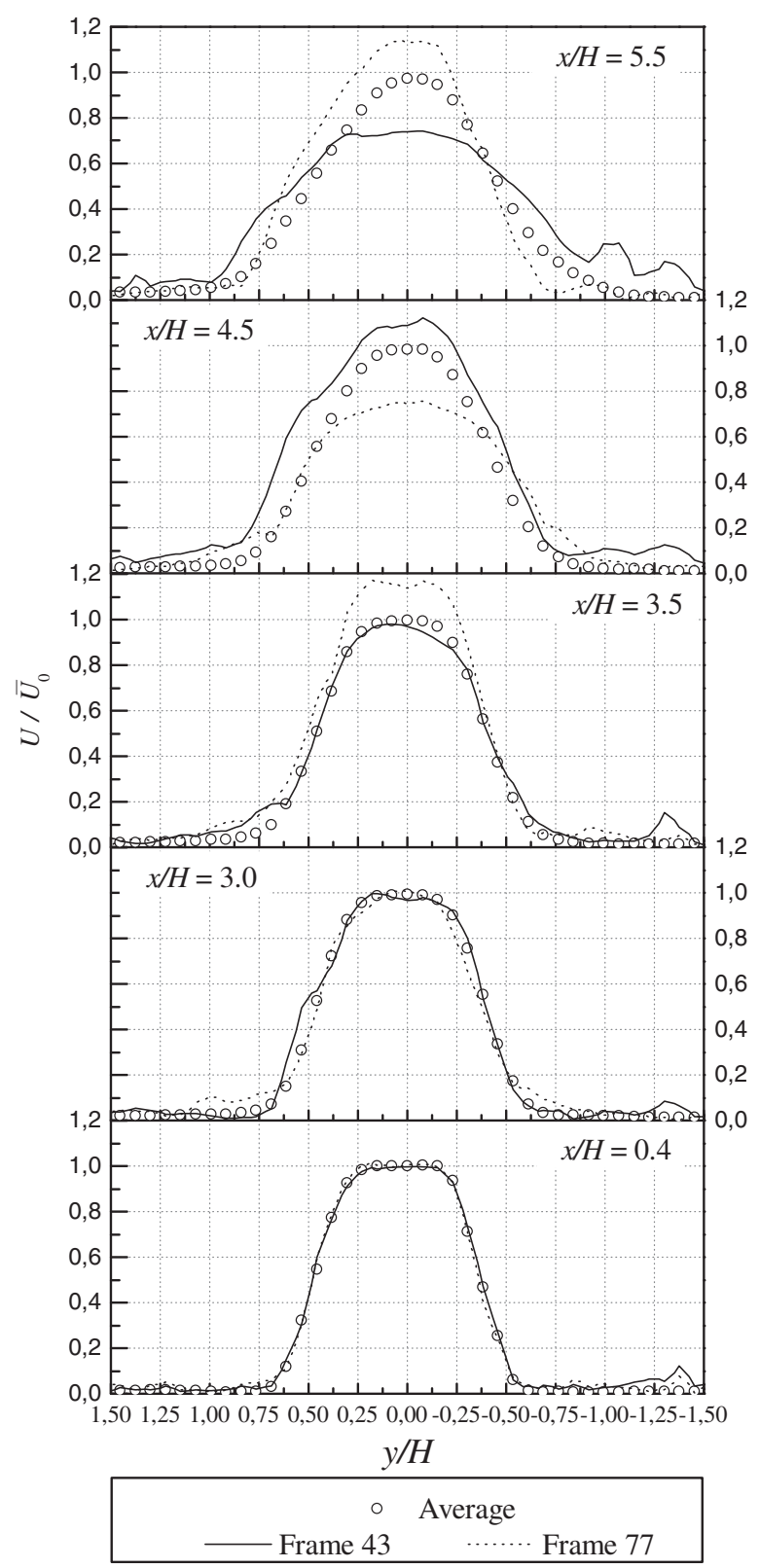

Fig. 9. Instant PIV velocity measurements for $\mathrm{Re}=6800$.

as the total lengths, $L_{3} / H=5.2-6.2$, which are in agreement with the average values of Gori et al. (2013), $L_{P} / H=5-6$.

3.1.3.2. PIV measurements. Fig. $6 \mathrm{c}$ presents the instant dimensionless centerline velocity measurements, $U / \bar{U}_{0}$, along with the average ones, as 100 frames, versus the distance $x / H$ from the jet exit. The variations of the dimensionless centerline velocity, $U / \bar{U}_{0}$, of the two frames are within $\pm 4 \%$ up to $x / H=2.7$, identifying the length of the flow $\mathrm{F}_{\mathrm{CIV}}, L_{C I V} / H=2.7$. After $L_{C I V}$, frame 99 presents a maximum in the height contraction, $x / H=3.6$, and a minimum in the height enlargement, $x / H=4.5$, where velocity on the centerline decreases of more than $20 \%$, allowing the pressure to increase, according to the Bernoulli equation, and to expel the fluid from the core of the jet to form the vortex. Frame 23 shows two maximums in the two height contractions, $x / H=3.0$ and $x / H=4.7$, and two minimums in the height enlargements, $x / H=3.7$ and $x / H=5.5$. In correspondence with each minimum of the velocity the pressure increases, according to the Bernoulli equation, expelling the fluid from the core jet to form the vortex.
Fig. $6 \mathrm{~d}$ presents the instant turbulence intensity measurements, $T u$, on the centerline. Turbulence intensity remains below $T u=4.0 \%$ up to $x / H=2.7$ for both frames, i.e. $L_{C I T} / H=2.7$. Turbulence intensity in the flow $\mathrm{F}_{\mathrm{SVT}}$ increases, but remaining below $T u=5.0 \%$ up to $x / H=3.3$ for frame 99 and up to $x / H=2.8$ for frame 23 . The sum of the lengths of the flows $\mathrm{F}_{\mathrm{CIT}}$ and $\mathrm{F}_{\mathrm{SVT}}$ is then $\left(L_{C I T}+L_{S V T}\right) / H=3.3$ for frame 99 and $\left(L_{C I T}+L_{S V T}\right) / H=2.8$ for frame 23. Turbulence intensity of frame 99 presents a greater spike at $x / H=3.5$, i.e. before the first vortex. Turbulence intensity of frame 23 presents a greater spike at $x / H=3.2$, i.e. before the first vortex.

The lengths of the flows $\mathrm{F}_{\mathrm{CIV}}, L_{\mathrm{CIV}} / H=2.7$, and $\mathrm{F}_{\mathrm{CIT}}, L_{\mathrm{CIT}} / H=2.7$, are reported in Table 1 showing they are in agreement with the length of the flow $\mathrm{F}_{\mathrm{ClH}}, L_{\mathrm{ClH}} / \mathrm{H}=2.5$, and the average values of Gori et al. (2013), $L_{C T} / H=2-3$. The length $\left(L_{C I T}+L_{S V T}\right) / H=3.3$ of frame 99 is comparable to the length $\left(L_{C I H}+L_{S V H}\right) / H=3.2$ of the same frame, while the length $\left(L_{C I T}+L_{S V T}\right) / H=2.8$ of frame 23 is equal to $\left(L_{C I H}+L_{S V H}\right) / H=2.8$ of the same frame. The length $\left(L_{C I T}+L_{S V T}\right) / H$ is shorter for frame 23 due to the presence of the second vortex.

Fig. 7 presents the dimensionless instant velocity measurements, $U / \bar{U}_{0}$, versus the transverse dimensionless distance, $y / H$, at five downstream distances from the slot exit, corresponding to the marks of Fig. $6 a$ and $b$. The first distance, $x / H=0.4$, is the closest to the exit and the instant measurements of frames 99 and 23 are in agreement with the average ones. The two instant measurements at the second distance, $x / H=2.5$, are practically unchanged compared to the exit values at $x / H=0.4$, marking the end of the flow $\mathrm{F}_{\mathrm{UVP}}$, at $L_{U V P} / H=2.5$. At the third distance, $x / H=3.7$, i.e. inside the flow with Coherent Vortices, $\mathrm{F}_{\mathrm{CV}}$, frame 99 shows a velocity increase because of the height contraction, while frame 23 presents a velocity decrease because of the height enlargement. At the fourth distance, $x / H=4.7$, still inside the flow $\mathrm{F}_{\mathrm{CV}}$, frame 99 shows a velocity decrease due to the height enlargement, while frame 23 presents a velocity increase, due to the height contraction. The last distance, $x / H=5.2$, marks the end of the flow $\mathrm{F}_{\mathrm{CV}}$ for frame $99, L_{3} / H=5.2$.

The length of the flow with Unchanged Velocity Profile, $F_{U V P}$, $L_{U V P} / H=2.5$, is reported in Table 1 , and is in agreement with the lengths $L_{C I H} / H=2.5, L_{C I V} / H=L_{C I T} / H=2.7$, and the average values of Gori et al. (2013), $L_{U} / H=2-3$.

\subsection{4. $R e=6,800$}

3.1.4.1. Visual inspection. The two instant visualizations at $\mathrm{Re}=6800$ are reported in Fig. 8a for frame 43 with one vortex, and in Fig. 8b for frame 77 with two vortices. The centerline instant velocity and turbulence measurements are in Fig. $8 \mathrm{c}$ and d. Fig. 8a and $b$ show also, as dashed line, the average flow which envelopes correctly the instant visualizations. In Fig. 8a and b the five marks indicate the positions of the instant velocity profile measurements.

Frame 43 shows the flow $\mathrm{F}_{\mathrm{CIH}}$ of length $L_{\mathrm{CIH}} / H=2.9$, the flow $\mathrm{F}_{\mathrm{SVH}}$ which extends up to $x / H=4.2$, i.e. $\left(L_{\mathrm{ClH}}+L_{S V H}\right) / H=4.2$, followed by a large vortex and by the vortices breakdown. The end of the vortex is at $x / H=6.5$, then the total length is $L_{3} / H=6.5$, before the vortices breakdown. Frame 77 shows the flow $\mathrm{F}_{\mathrm{CIH}}$ of length $L_{C I H} / H=2.9$, followed by the flow $\mathrm{F}_{\mathrm{SVH}}$ up to $x / H=3.4$, i.e. $\left(L_{C I H}+L_{S V H}\right) / H=3.4$. The flow $\mathrm{F}_{\mathrm{SVH}}$ is longer for frame 43 because only one vortex is present. The end of the second vortex at $x / H=7.0$ marks the total length for frame $77, L_{3} / H=7.0$, before the vortices breakdown.

The results of the visual inspection, $L_{C I H} / H,\left(L_{C I H}+L_{S V H}\right) / H$, and $L_{3} / H$, are reported in Table 1 . The length of the flow $\mathrm{F}_{\mathrm{CIH}}$, $L_{C I H} / H=2.9$, is in agreement with the average value of Gori et al. (2013), $L_{C H} / H=3.0$. The end of the coherent vortex flow for frame $43, L_{3} / H=6.5$, is slightly longer than the average value, $L_{P} / H=6.0$, while the end of the coherent vortex flow for frame 77 , $L_{3} / H=7.0$, is longer than the average one. 

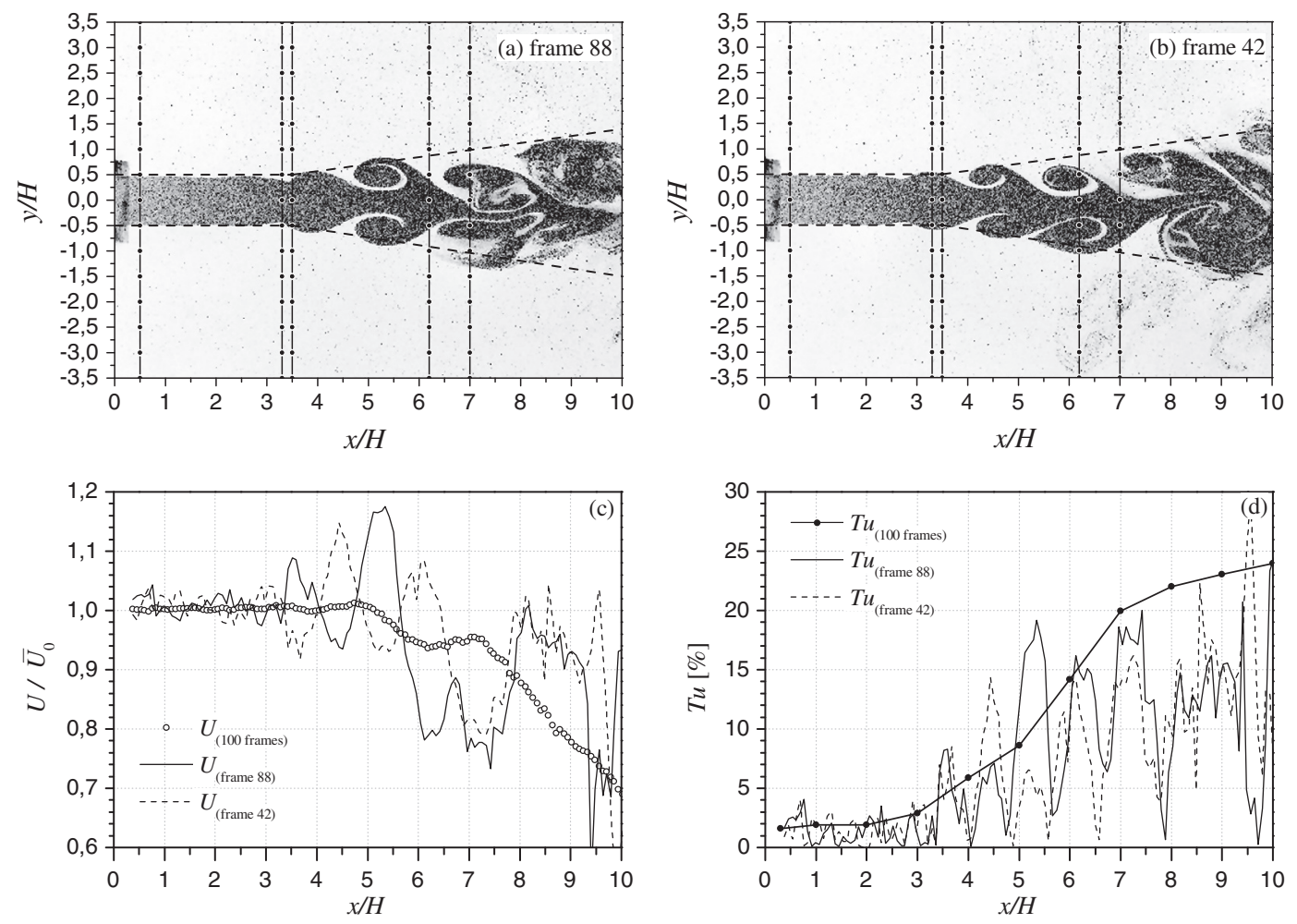

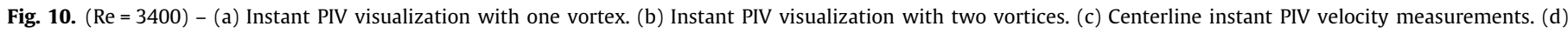
Centerline instant PIV turbulence intensity measurements.

3.1.4.2. PIV measurements. Fig. 8c presents the instant dimensionless centerline velocity measurements, $U / \bar{U}_{0}$, along with the average ones, versus the distance $x / H$ from the jet exit. The dimensionless centerline velocities, $U / \bar{U}_{0}$, of the two frames are within $\pm 4 \%$ up to $x / H=3.3$, identifying the length of the flow $\mathrm{F}_{\mathrm{CIV}}$, $L_{C I V} / H=3.3$. After $L_{C I V}$, frame 43 presents a maximum in the height contraction, $x / H=4.5$, and a minimum in the height enlargement, $x / H=5.5$, where velocity on the centerline decreases of more than $40 \%$, allowing the pressure to increase, according to the Bernoulli equation, and to expel the fluid from the jet core to form the vortex. Frame 77 shows two maximums in the two height contractions, $x / H=3.5$ and $x / H=5.5$, and two large minimums in the height enlargements, $x / H=4.5$ and $x / H=6.5$. In correspondence with each minimum of the velocity the pressure increases, according to the Bernoulli equation, and the jet expels the fluid from the core to form the vortices, the first one at $x / H=4.5$ and the second one at $x / H=6.5$.

Fig. $8 \mathrm{~d}$ presents the instant turbulence intensity measurements on the centerline. Turbulence intensity of both frames remains below $T u=4.0 \%$ up to $x / H=3.2$, i.e. $L_{C I T} / H=3.2$. Turbulence intensity increases but remaining below $\mathrm{Tu}=5.0 \%$ up to $x / H=4.3$ for frame 43 , and to $x / H=3.3$ for frame 77 , which correspond to the beginning of the first vortex. The total length of the flows $\mathrm{F}_{\mathrm{CIT}}$ and $\mathrm{F}_{\mathrm{SVT}}$ is $\left(L_{C I T}+L_{S V T}\right) / H=4.3$ for frame 43 and $\left(L_{C I T}+L_{S V T}\right) / H=3.3$ for frame 77 . Turbulence intensity of frame 43 presents a large spike of turbulence at $x / H=5.6$, in correspondence of the vortex. Turbulence intensity of frame 77 presents a spike of $T u=15 \%$ at $x / H=3.5$, before the first vortex.

The length of the flow $\mathrm{F}_{\mathrm{CIT}}$ for the two frames are reported in Table 1 showing that the length $L_{C I T} / H=3.2$ is in agreement with $L_{C I V} / H=3.3$, and only slightly longer than the instant length $L_{C I H} / H=2.9$, and the average value of Gori et al. (2013), $L_{C H} / H=3$. Further on, the length $\left(L_{C I T}+L_{S V T}\right) / H=4.3$ is in agreement with the length $\left(L_{C I H}+L_{S V H}\right) / H=4.2$ for frame 43 and the length $\left(L_{C I T}+L_{S V T}\right) / H=3.3$ with the length $\left(L_{C I H}+L_{S V H}\right) / H=3.4$ for frame 77 .
Fig. 9 presents the dimensionless instant velocity measurements, $U / \bar{U}_{0}$, versus the transverse dimensionless distance, $y / H$, at five downstream distances from the slot exit, corresponding to the marks of Fig. $8 \mathrm{a}$ and $\mathrm{b}$. The first distance, $x / H=0.4$, is the closest to the exit and the two instant measurements of frames 43 and 77 are in agreement with the average one. The two instant measurements at the second distance, $x / H=3.0$, are practically unchanged compared to the exit values at $x / H=0.4$, marking the end of the flow $\mathrm{F}_{\mathrm{UVP}}$, at $L_{U V P} / H=3.0$. At the third distance, $x / H=3.5$, i.e. in the flow $F_{S V H}$, the instant velocity profile of frame 43 is in agreement with the average one because the height of the jet is equal to the exit one, while frame 77 presents an instant velocity profile greater than the average one because of the height contraction. At the fourth distance, $x / H=4.5$, i.e. inside the vortex region, frame 43 shows a velocity increase over the average, due to the height contraction, while frame 77 presents a velocity decrease because of the height enlargement. At the fifth distance, $x / H=5.5$, it happens the opposite for similar reasons.

The length of the flow with Unchanged Velocity Profile, Fuv, $L_{U V P} / H=3.0$, is reported in Table 1 , and is in agreement with the lengths $L_{C I H} / H=2.9, L_{C I V} / H=3.3, L_{C I T} / H=3.2$, and the average one of Gori et al. (2013), $L_{U} / H=3.0$.

\subsection{5. $R e=3400$}

3.1.5.1. Visual inspection. The two instant visualizations at $\mathrm{Re}=3400$ are reported in Fig. 10a for frame 88 with one vortex, and in Fig. 10b for frame 42 with two vortices. The centerline instant velocity and turbulence measurements are in Fig. 10c and d. Fig. 10a and b show, as dashed line, the average flow, which envelopes correctly the instant visualizations. In Fig. 10a and b the five marks indicate the positions of the instant velocity profile measurements.

Frame 88 shows the flow $\mathrm{F}_{\mathrm{CIH}}$ long $L_{\mathrm{CIH}} / H=3.1$, the flow $\mathrm{F}_{\mathrm{SVH}}$ which extends up to $x / H=4.8$, i.e. $\left(L_{C I H}+L_{S V H}\right) / H=4.8$, followed by a large vortex, ending at $x / H=7.0$, i.e. the total length is 


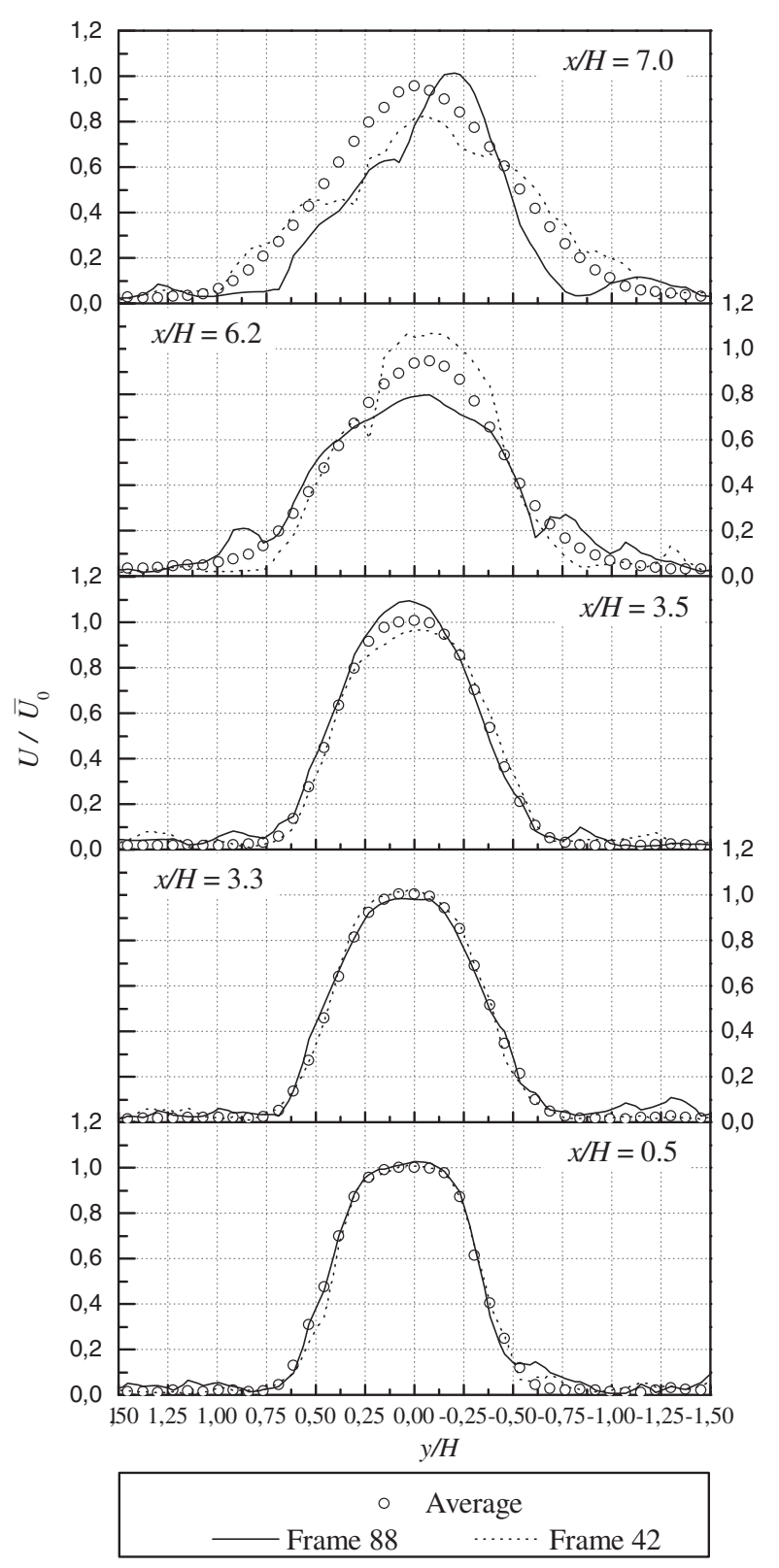

Fig. 11. Instant PIV velocity measurements for $\mathrm{Re}=3400$.

$L_{3} / H=7.0$, and by the vortices breakdown. Frame 42 shows the flow $\mathrm{F}_{\mathrm{CIH}}$ of length $L_{\mathrm{CIH}} / H=2.9$, followed by the flow $\mathrm{F}_{\mathrm{SVH}}$ up to $x / H=4.2$, i.e. $\left(L_{C I H}+L_{S V H}\right) / H=4.2$. The end of the second vortex at $x / H=7.5$ marks the total length, $L_{3} / H=7.5$.

The results of the visual inspection, $L_{C I H} / H,\left(L_{C I H}+L_{S V H}\right) / H$, and $L_{3} / H$, are reported in Table 1 . The lengths of the flow $\mathrm{F}_{\mathrm{CIH}}$ for the two frames, $L_{C I H} / H=3.1-2.9$, are in the lower part of the average values of Gori et al. (2013), $L_{C I H} / H=3-4$. The end of the coherent vortices flow for frame $88, L_{3} / H=7.0$, is in agreement with the average value, $L_{P} / H=7.0$, while the length for frame 42 , $L_{3} / H=7.5$, is slightly longer.

3.1.5.2. PIV measurements. Fig. 10c presents the instant dimensionless centerline velocity measurements, $U / \bar{U}_{0}$, along with the average ones, versus the distance $x / H$ from the jet exit. The dimensionless centerline velocities, $U / \bar{U}_{0}$, of the two frames are within $\pm 4 \%$ up to $x / H=3.4$, identifying the length of the flow $\mathrm{F}_{\mathrm{CIV}}$, $L_{C I V} / H=3.4$. After $L_{C I V}$, frame 88 , with one vortex, has two maximums in the height contractions, $x / H=3.5$ and $x / H=5.3$, and two minimums in the height enlargements, $x / H=4.3$ and $x / H=6.1$. At $x / H=6.1$ the velocity on the centerline decreases more than $20 \%$, allowing the pressure to increase, according to the Bernoulli equation, and to expel the fluid from the jet core to form the vortex. Frame 42, with two vortices, shows three minimums of the velocity in the height enlargements, $x / H=3.6, x / H=5.2$ and $x / H=7.0$, and two maximums in the height contractions, $x / H=4.5$ and $x / H=6.1$. The minimums of the velocity at $x / H=5.1$ and $x / H=7.0$ allow the formation of the two vortices.

Fig. 10d presents the instant turbulence intensity measurements on the centerline. Turbulence intensity variations maintain below $\mathrm{Tu}=4 \%$ up to $x / H=3.4$ for both frames, i.e. $L_{C I T} / H=3.4$. Turbulence intensity increases, but remaining below $\mathrm{Tu}=9 \%$, up to $x / H=4.8$ for frame 88 and up to $x / H=4.2$ for frame 42 , both corresponding to the beginning of the first vortex. The sum of the lengths of the flows $\mathrm{F}_{\mathrm{CIT}}$ and $\mathrm{F}_{\mathrm{SVT}}$ is then $\left(L_{C I T}+L_{S V T}\right) / H=4.8$ for frame 88 and $\left(L_{C I T}+L_{S V T}\right) / H=4.2$ for frame 42 . Turbulence intensity of frame 88 presents a spike of turbulence at $x / H=5.1$, in correspondence with the vortex. Turbulence intensity of frame 42 presents a spike of turbulence at $x / H=4.5$, in correspondence with the first vortex, and a second spike at $x / H=6.2$, in correspondence with the second vortex.

The length of the flow $\mathrm{F}_{\mathrm{CIT}}$ for the two frames is reported in Table 1 showing that the length $L_{C I T} / H=3.4$ is equal to $L_{C I V} / H$ and only slightly longer than $L_{C I H} / H=3.1-2.9$. The length $L_{C I T} / H$ is also in agreement with the average values of Gori et al. (2013), $L_{U} / H=3-4$. Further on, the length $\left(L_{C I T}+L_{S V T}\right) / H=4.8$ is equal to the length $\left(L_{C I H}+L_{S V H}\right) / H$ for frame 88 , and $\left(L_{C I T}+L_{S V T}\right) / H=4.2$ is equal to the length $\left(L_{C I H}+L_{S V H}\right) / H$ for frame 42 .

Fig. 11 presents the dimensionless instant velocity measurements, $U / \bar{U}_{0}$, versus the transverse dimensionless distance, $y / H$, at five downstream distances from the slot exit, corresponding to the marks of Fig. $10 \mathrm{a}$ and $\mathrm{b}$. The first distance, $x / H=0.5$, is the closest to the exit and the two instant measurements of frames 88 and 42 are in agreement with the average one. The two instant measurements at the second distance, $x / H=3.3$, are practically unchanged compared to the values at $x / H=0.5$, marking the end of the flow $\mathrm{F}_{U V P}$, at $L_{U V P} / H=3.3$. At the third distance, $x / H=3.5$, i.e. inside the flow $\mathrm{F}_{\mathrm{SVH}}$, frame 88 shows a velocity greater than the average one because of the height contraction, while frame 42 presents a velocity decrease because of the height enlargement. At the fourth distance, $x / H=6.2$, i.e. inside the flow $\mathrm{F}_{\mathrm{CV}}$, frame 42 shows a velocity increase over the average due to the height contraction, while frame 88 presents a velocity decrease because of the height enlargement. At the fifth distance, $x / H=7.0$, the maximum velocity of frame 88 is equal to the centerline average velocity but the velocity profile has a shift towards the lower part of the jet, as shown by Fig. 10b, on the right, while the maximum velocity of frame 42 is smaller than the maximum average velocity because of the height enlargement.

The length of the flow with Unchanged Velocity Profile, $\mathrm{F}_{\mathrm{UVP}}$, $L_{U V P} / H=3.3$, reported in Table 1 , is in fair agreement with the lengths $L_{C I H} / H=3.1-2.9, L_{C I V} / H=3.4, L_{C I T} / H=3.4$, and slightly smaller than the average value of Gori et al. (2013), $L_{U} / H=4.0$.

\subsection{Flow in the upper laminar regime}

\subsection{1. $R e=2200$}

3.2.1.1. Visual inspection. The two instant visualizations in the upper laminar regime, $\operatorname{Re}=2200$, are reported in Fig. 12a for frame 22 with one vortex, and in Fig. 12b for frame 31 with two vortices, while the centerline instant velocity and turbulence are in Fig. 12c and d. Fig. 12a and b shows the average flow which envelopes correctly the instant visualizations. The five marks of Fig. 12a and b indicate the positions of the instant velocity measurements. 

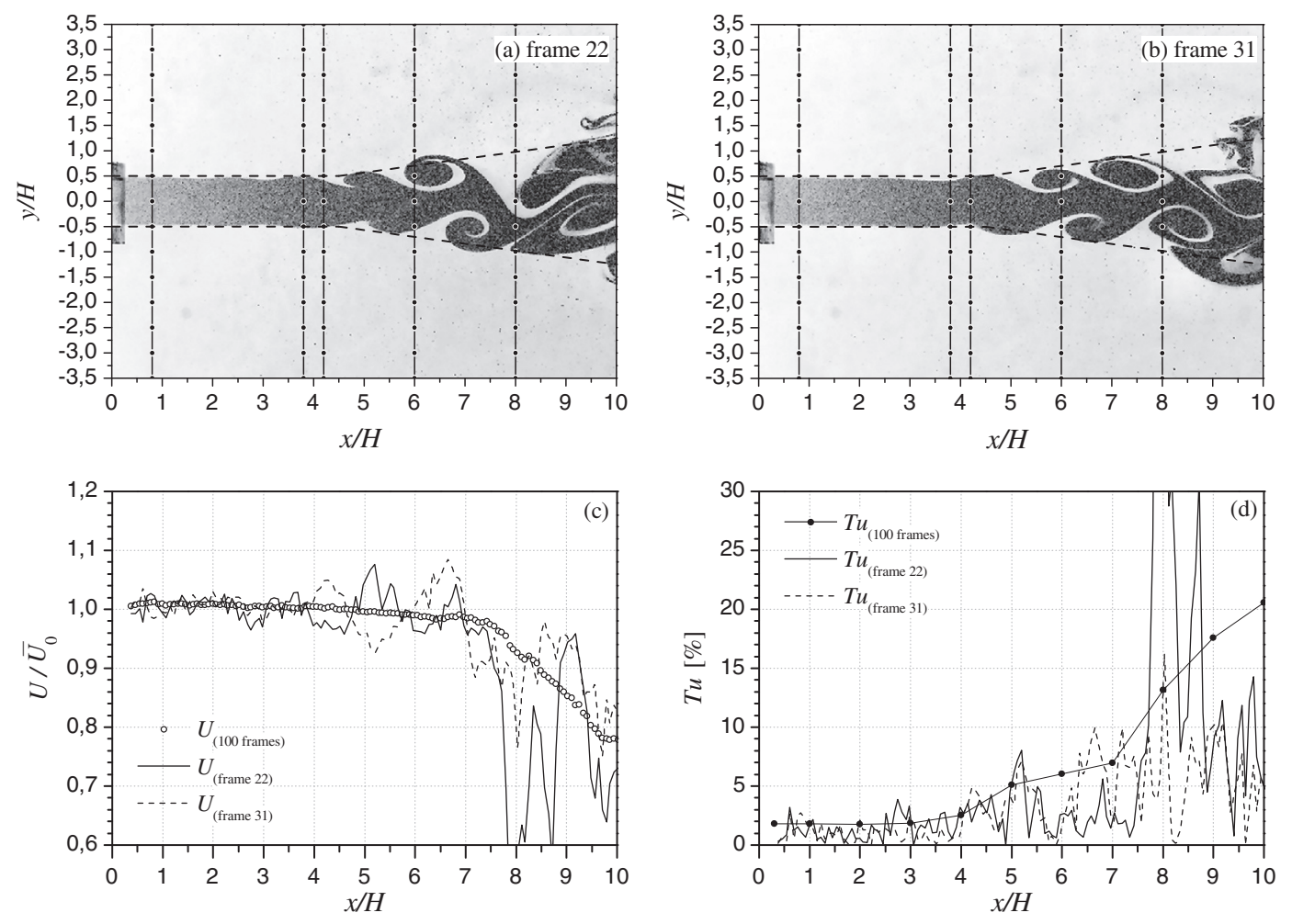

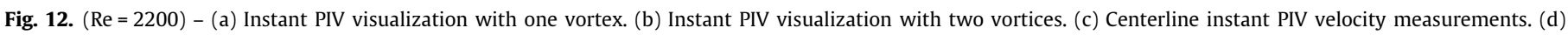
Centerline instant PIV turbulence intensity measurements.

Frame 22 of Fig. 12a shows the flow $\mathrm{F}_{\mathrm{CIH}}$ of length $L_{\mathrm{CIH}} / H=4.0$, and the flow $\mathrm{F}_{\mathrm{SVH}}$ up to $\left(L_{\mathrm{CIH}}+L_{\mathrm{SVH}}\right) / H=5.9$. The end of the flow $\mathrm{F}_{\mathrm{CV}}$ is at $x / H=8.0$, i.e. the total length is $L_{3} / H=8.0$, followed by the vortices breakdown. Frame 31 of Fig. 12b shows the flow $\mathrm{F}_{\mathrm{CIH}}$ of length $L_{C I H} / H=4.0$, followed by the flow $\mathrm{F}_{\mathrm{SVH}}$ up to $\left(L_{\mathrm{CIH}}+L_{S V H}\right) / H=5.5$. The flow $\mathrm{F}_{\mathrm{SVH}}$ is longer in the frame with one vortex. The end of the second vortex at $x / H=9.0$ marks the total length, $L_{3} / H=9.0$.

The lengths of the visual inspection, $L_{C I H} / H,\left(L_{C I H}+L_{S V H}\right) / H$, and $L_{3} / H$, are reported in Table 1 . The length of the flow $\mathrm{F}_{\mathrm{CIH}}$, $L_{C I H} / H=4.0$, is in the lower part of the average values of Gori et al. (2013), $L_{U} / H=4-5$. The end of the coherent vortices for frame 22 , $L_{3} / H=8.0$, is in the upper part of the average values, $L_{P} / H=7-8$, while the length for frame $31, L_{3} / H=9.0$, is longer than the average ones.

3.2.1.2. PIV measurements. Fig. $12 \mathrm{c}$ presents the instant PIV dimensionless centerline velocity measurements, $U / \bar{U}_{0}$, along with the average ones of Gori et al. (2013), versus the distance $x / H$ from the jet exit. The variations of the dimensionless centerline velocities, $U / \bar{U}_{0}$, of the two frames are within $\pm 4 \%$ up to $x / H=4.1$, marking the length of the flow $\mathrm{F}_{\mathrm{CIV}}, L_{C I V} / H=4$.1. After $L_{C I V}$, velocity starts oscillating around the average one at a greater rate. Frame 22, with one vortex, has two maximums in the height contractions, $x / H=5.1$ and $x / H=6.8$, and only small minimums. At $x / H=7.9$ the decrease of the velocity reaches $50 \%$, which is responsible for the pressure increase and the vortex formation. Frame 31, with two vortices, shows two maximums of the velocity in the height contractions, $x / H=4.2$ and $x / H=6.5$, and two minimums in the height enlargements, $x / H=5.1$ and $x / H=7.2$. The velocity variation at $x / H=5.1$ is equal to $-6 \%$, i.e. it is only slightly greater than in the previous flow, and the relative variation of the pressure is only able of increasing the height of jet. The variation of velocity in $x / H=7.2$ is greater, around $-12 \%$, and the correspondent pressure variation is able to expel the fluid to form the vortex.
Fig. 12d presents the instant PIV turbulence intensity on the centerline for frames 22 and 31, compared to the average ones. Turbulence intensity of both frames remains smaller than $4.0 \%$ up to $x / H=4.1$, giving a length of the flow $\mathrm{F}_{\mathrm{CIT}}, L_{C I T} / H=4.1$. Turbulence intensity of both frames increases, but remaining below $8.0 \%$ up to $x / H=5.2$, with a length $\left(L_{C I T}+L_{S V T}\right) / H=5.2$. Turbulence intensity of frame 22 presents a large spike of $T u=60 \%$ at $x / H=8$, in correspondence of the vortex. Frame 31 presents smaller spikes on the centerline because the jet is more central.

The length of the flow $\mathrm{F}_{\mathrm{CIT}}$ for the two frames is reported in Table 1 , showing that $L_{C I T} / H=4.1$ is equal to $L_{C I V} / H$ and slightly longer than $L_{\mathrm{CIH}} / H=4.0$. They are in agreement also with the average values of Gori et al. (2013), $L_{C T} / H=4-5$.

Fig. 13 presents the dimensionless instant velocity measurements, $U / \bar{U}_{0}$, versus the transverse dimensionless distance, $y / H$, at five downstream distances from the slot exit, corresponding to the marks of Fig. 12a and b. The first distance, $x / H=0.8$, is the closest to the exit and its profile is assumed as the exit one. At $x / H=0.8$, the two instant measurements of frames 22 and 31 are in agreement with the average one in the jet region. The two instant measurements at the second distance, $x / H=3.8$, are practically unchanged compared to the exit. At the third distance, $x / H=4.2$, the profiles of the two frames are only slightly different on the centerline, marking the end of the flow $F_{U V P}$, at about $L_{U V P} / H=4.1$, in agreement with the length of the flow $\mathrm{F}_{\mathrm{CIV}}$, $L_{C I V} / H=4.1$. At the fourth distance, $x / H=6.0$, the two instant velocity profiles are moved from the centerline, but remaining with a similar value on the centerline and thus justifying the average values of Gori et al. (2013), $L_{U} / H=6$. At the fifth distance, $x / H=8.0$, the maximum of frame 22 has moved away from the centerline and is smaller than the average one, marking the end of the flow $\mathrm{F}_{\mathrm{CV}}, L_{3} / H=8.0$. The maximum velocity of frame 31 is quite similar to the average one because the flow is still in the coherent vortices flow, $\mathrm{F}_{\mathrm{CV}}$. 


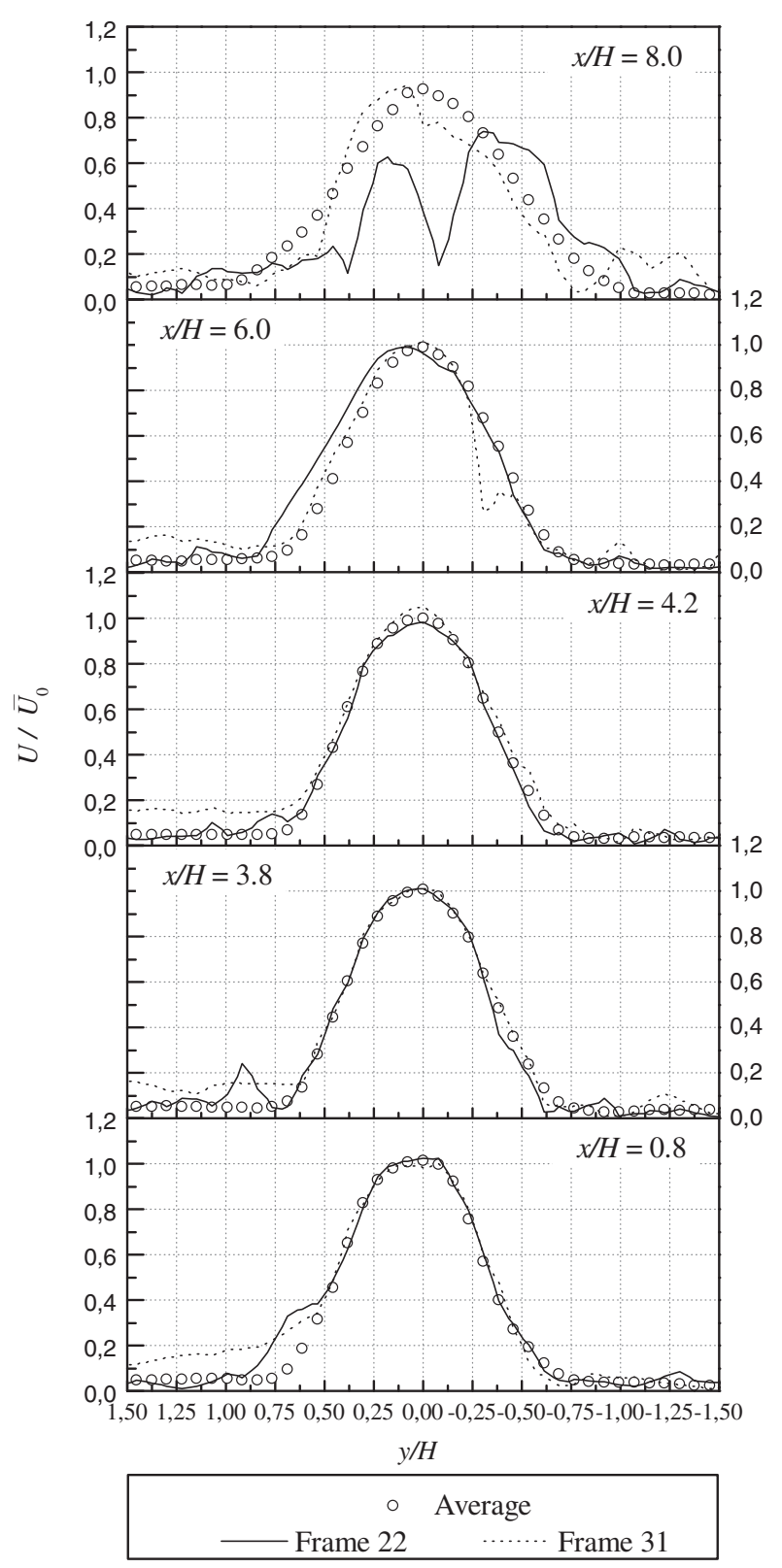

Fig. 13. Instant PIV velocity measurements for $\mathrm{Re}=2200$.

The length of the flow $\mathrm{F}_{\mathrm{UVP}}, L_{U V P} / H=4.1$, is reported in Table 1 and is in fair agreement with the lengths $L_{C I H} / H=4.0$, $L_{C I V} / H=L_{C I T} / H=4.1$.

\subsection{Lengths of the instant types of flow}

Table 1 presents the lengths of the instant flows detected for the six Reynolds numbers investigated, reported in the first column.

The columns from the second to the fifth one report the length ratios of the flows with Constant Instant Height $\mathrm{F}_{\mathrm{ClH}}, L_{\mathrm{ClH}} / \mathrm{H}$, with Constant Instant Velocity on the centerline $\mathrm{F}_{\mathrm{CIV}}, L_{\mathrm{CIV}} / \mathrm{H}$, with Constant Instant Turbulence on the centerline $\mathrm{F}_{\mathrm{CIT}}, L_{\mathrm{CIT}} / \mathrm{H}$, and with Unchanged Velocity Profile $F_{U V P}, L_{U V P} / H$. The length ratio of the flow $\mathrm{F}_{\mathrm{CIH}}, L_{\mathrm{CIH}} / H$, is slightly greater for the frame with one vortex at $\operatorname{Re}=3400$, while the other ratios $L_{C I V} / H, L_{C I T} / H, L_{U V P} / H$, are independent on the number of vortices. The four ratios $L_{C I H} / H, L_{C I V} / H, L_{C I T} / H$, $L_{U V P} / H$, are practically equal and they increase with the decrease of the Reynolds number, without discontinuity between turbulent and laminar regimes.
The four types of flow are considered due to the same phenomenon because the fluctuations of velocity and turbulence on the centerline are smaller than $\pm 4 \%$ and do not alter the jet height, and the relative flow is called flow with Negligible Disturbances, $\mathrm{F}_{\mathrm{ND}}$. The four lengths, i.e. $L_{C I H} \sim L_{C I V} \sim L_{C I T} \sim L_{U V P}$, are then relative to the flow with Negligible Disturbances, $\mathrm{F}_{\mathrm{ND}}$, or first type of flow, $L_{N D}=L_{1}$. The length ratios of the flow with Negligible Disturbances, $L_{N D} / H=L_{1} / H$, are reported in the sixth column of Table 1 as average of the four lengths, $L_{C I H} / H, L_{C I V} / H, L_{C I T} / H, L_{U V P} / H$.

The flow with Small Variations of the Height, $\mathrm{F}_{\mathrm{SVH}}$, of length $L_{S V H}$, and the flow with Small Variations of the Turbulence, $F_{S V T}$, of length $L_{S V T}$, are present after the flow $\mathrm{F}_{\mathrm{ND}}$. The variations of the turbulence intensity in $\mathrm{F}_{\mathrm{SVT}}$ are smaller than 5\% from $\mathrm{Re}=35,300$ to $\operatorname{Re}=6800$, increasing only at $8-9 \%$ for $\operatorname{Re}=3400$ and 2200 . The lengths $\left(L_{C I H}+L_{S V H}\right)$ and $\left(L_{C I T}+L_{S V T}\right)$ are almost equal, and greater for the frame with one vortex, opposite to what happens for the flow with Constant Instant Height $\mathrm{F}_{\mathrm{CIH}}$. The two flows $\mathrm{F}_{\mathrm{SVH}}$ and $\mathrm{F}_{\mathrm{SVT}}$ are due to the same phenomenon of small disturbances, and are called flow with Small Disturbances, $\mathrm{F}_{\mathrm{SD}}$, or second type of flow, with length $L_{S D}$. The total length $\left(L_{C I H}+L_{S V H}\right) \sim\left(L_{C I T}+L_{S V T}\right)$ increases with the decrease of the Reynolds number, without discontinuity between turbulent and laminar regime, it is equal to $\left(L_{N D}+L_{S D}\right)=$ $L_{2}$, and its average is reported in the ninth column of Table 1 as $L_{2} / H$, where $L_{2}$ is the average of the sum of the lengths of the flows with negligible and small disturbances.

After $L_{2}$, velocity and turbulence fluctuations are greater inducing large disturbances of the pressure, which are able to expel the fluid from the core of the jet to form a coherent vortex. The flow with Coherent Vortices, $\mathrm{F}_{\mathrm{CV}}$, or third type of flow, has a length $L_{C V}$, after which starts the vortex breakdown, or fourth type of flow. The sum of the lengths of the three flows $\left(L_{N D}+L_{S D}+L_{C V}\right)=L_{3}$, is longer for the frame with two vortices, as in the flow $\mathrm{F}_{\mathrm{ClH}}$, and increases with the decrease of the Reynolds number. The length ratios $L_{3} / H$, for the two vortices are reported in the last column of Table 1 , as average one of the two frames.

Fig. 14 presents, as continuous line, the instant length ratio of the flow $\mathrm{F}_{\mathrm{ND}}$, or first type of flow, $L_{N D} / H=L_{1} / H$, and the length ratios $L_{2} / H$, and $L_{3} / H$, in order to have a graphical impression. The length of the flow $\mathrm{F}_{\mathrm{ND}}, L_{1} / H$, increases with the decrease of the Reynolds number, especially in the upper laminar regime. The length ratio $L_{2} / H$, sum of the length ratios $L_{N D} / H$ and $L_{S D} / H$, has a similar trend. It can be noticed that the length ratio of the flow $\mathrm{F}_{\mathrm{SD}}, L_{S D} / H=$ $L_{2} / H-L_{1} / H$, has values in the range $L_{S D} / H=0.6-1.7$, which is only slightly increasing with the decrease of the Reynolds number. The length ratio $L_{3} / H$, sum of $L_{N D} / H, L_{S D} / H$ and $L_{C V} / H$, is practically parallel to $L_{2} / H$, i.e. the length ratio of the flow $\mathrm{F}_{\mathrm{CV}}, L_{C V} / H=L_{3} / H-L_{2} / H$, maintains almost constant in the range of the Reynolds number investigated, with values in the range $L_{C V} / H=2.6-3.2$.

The three instant length ratios, $L_{1} / H, L_{2} / H$, and $L_{3} / H$, are compared with the average values of Gori et al. (2013), $L_{C H} / H=L_{C T} / H$, $L_{U} / H$, and $L_{P} / H$, reported as dotted lines in Fig. 14. The average length ratios $L_{C H} / H$ and $L_{U} / H$ are similar in the turbulent regime

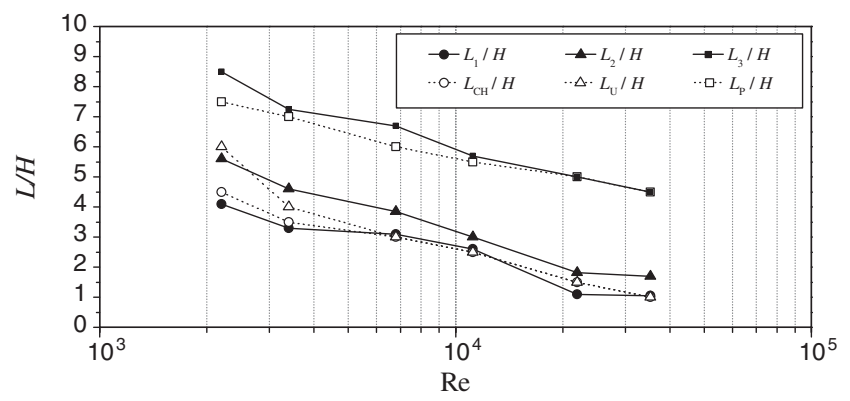

Fig. 14. Lengths of the different types of flow in turbulent and laminar regime. 


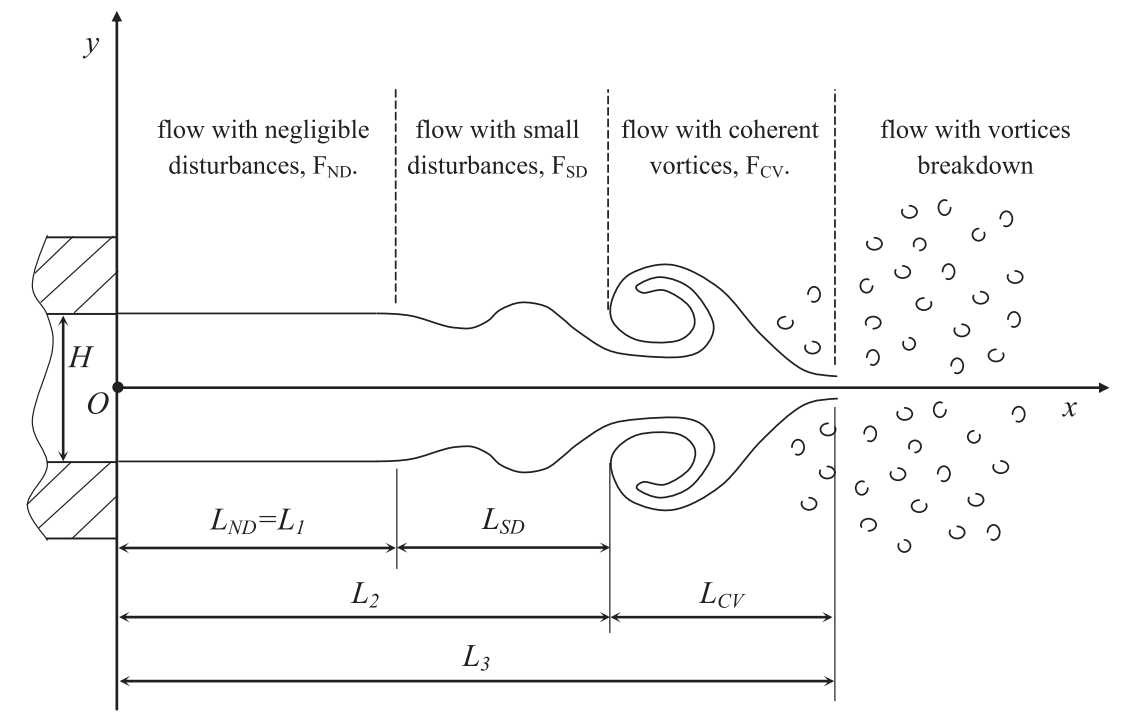

Fig. 15. Instant flow evolution of the jet.

while, in the upper laminar one, they are different because the average images are not able to detect the small lateral oscillations of the laminar jet at $\mathrm{Re}=2200$. The length ratio of the flow $\mathrm{F}_{\mathrm{ND}}$, or first type of flow, $L_{1} / H$, agrees with the average length ratios $L_{C H} / H$ and $L_{U} / H$ in turbulent flow, while the ratio $L_{2} / H$, not evidenced by the average visualizations, is in agreement with the average ratio $L_{U} / H$ only in the upper laminar flow. The instant length ratio $L_{3} / H$ agrees with the average ratio of the potential core, $L_{P} / H$, in the entire range of the Reynolds numbers experimented, i.e. from $\operatorname{Re}=2200$ to $\operatorname{Re}=35,300$.

Finally, Fig. 15 can represent the main conclusions of the instant flow evolution found in the present paper. The length of the flow with negligible disturbances $\mathrm{F}_{\mathrm{ND}}$ is $L_{1}=L_{N D}$, while $L_{2}$ is the sum of the lengths of the flows $\mathrm{F}_{\mathrm{ND}}$ and with small disturbances $\mathrm{F}_{\mathrm{SD}}, L_{N D}+L_{S D}$, and $L_{3}$ is the sum of the lengths of the flows $F_{N D}, F_{S D}$ and with coherent vortices $\mathrm{F}_{\mathrm{CV}}, L_{N D}+L_{S D}+L_{C V}$. The end of the flow $\mathrm{F}_{\mathrm{CV}}$ is at the length $L_{3}$, after which starts the vortices breakdown.

\section{Conclusions}

The main conclusion of the present paper is the update of the current state of art of the literature, as far as the instant flow evolution of rectangular turbulent free jets of air is concerned, in the Reynolds number range from $\operatorname{Re}=35,300$ to $\operatorname{Re}=2200$, thanks to the PIV visualizations and measurements.

Visual inspection of the instant PIV frames, with one vortex or two, show that, just after the exit, is present a type of flow where the height of the jet remains constant for a length $L_{\mathrm{CIH}}$, called flow with Constant Instant Height. PIV measurements of the instant centerline velocity and turbulence intensity along the jet development report variations smaller than $\pm 4 \%$ for a length $L_{C I V}$, called flow with Constant Instant Velocity on the centerline, and for a length $L_{C I T}$, called flow with Constant Instant Turbulence on the centerline, both in the turbulent and laminar regimes. PIV measurements of the instant velocity at several distances from the exit show that velocity profile remains practically constant for a length $L_{U V P}$, called flow with Unchanged Velocity Profile. Since the four lengths are quite similar, i.e. $L_{C I H} \sim L_{C I V} \sim L_{C I T} \sim L_{U V P}$, the relative flow is called flow with Negligible Disturbances, or first type of flow, with length $L_{C I H} \sim L_{C I V} \sim L_{C I T} \sim L_{U V P}=L_{N D}=L_{1}$.

After the flow with Negligible Disturbances, of length $L_{N D}=L_{1}$, visual inspection of the instant PIV frames shows small variations of the height, but without formation of vortex, and the relative flow is called flow with Small Variation of the Height, with a length $L_{S V H}$, greater for the images with one vortex. In this flow the instant PIV measurements report greater instant turbulence intensity variations, which are remaining below $5 \%$ from $\operatorname{Re}=35,300$ to $\operatorname{Re}=6800$, increasing at 8-9\% only for $\operatorname{Re}=3400-2200$, for a length $L_{S V T}$. The flow is called flow with Small Variations of the Turbulence. The flows with Small Variations of the Height or with Small Variations of the Turbulence are called flow with Small Disturbances, or second type of flow, and the two lengths $L_{S V H}$ and $L_{S V T}$ are then associated to the small disturbances, i.e. $L_{S D}$. In the second type of flow the small variations of velocity are due to the small variations of the height, because of the mass conservation, but without the vortex formation. The sum of the lengths of the two flows with Negligible and Small Disturbances, $\left(L_{N D}+L_{S D}\right)=L_{2}$, is the second length introduced in this paper, $L_{2}$.

After the flow with Small Disturbances the instant PIV visualizations show the flow with Coherent Vortices, after which starts the vortices breakdown. The instant PIV measurements in the flow with Coherent Vortices report greater variations of velocity and turbulence than in the previous two flows, inducing larger variations of the pressure, because of the Bernoulli equation, which are responsible for the fluid expulsion and the vortex formation, due to the lower velocity of the stagnant fluid outside the jet. The instant PIV visualizations allow defining the length of the flow with Coherent Vortices, $L_{C V}$, before the beginning of the vortices breakdown. The sum of the lengths of the flows with Negligible and Small Disturbances, and Coherent Vortices, $\left(L_{N D}+L_{S D}+L_{C V}\right)=L_{3}$, is the third length introduced in the paper, $L_{3}$.

The results of the present instant PIV visualizations compare favorably the length $L_{1}$ with the average lengths of the flow with Constant Height and Constant Turbulence $L_{C H} \sim L_{C T}$, the length $L_{2}$ with the average length of the Undisturbed region of Flow $L_{U}$ and the length $L_{3}$ with the average length of the Potential core $L_{P}$, previously measured by the same authors.

\section{References}

Abramovich, G.N., 1982. On the deformation of the rectangular turbulent jet crosssection. Int. J. Heat Mass Transf. 25 (12), 1885-1894.

Albertson, M.L., Dai, Y.B., Jensen, R.A., Rouse, H., 1948. Diffusion of submerged jets ASCE Paper 2409, vol. 74, pp. 1571-1596.

Bradbury, L.J.S., 1965. The structure of a self-preserving turbulent plane jet. J. Fluid Mech. 23 (1), 31-64.

Gogineni, S., Shih, C., 1997. Experimental investigation of the unsteady structure of a transitional plane wall jet. Exp. Fluids 23, 121-129. 
Goldstein, R.J., 1996. Fluid Mechanics Measurements. Taylor \& Francis, New York. Gori, F., Bossi, L., 2000a. On the cooling effect of an air jet along the surface of cylinder. Int. Commun. Heat Mass Transfer 27 (5), 667-676.

Gori, F., Bossi, L., 2000b. On the optimal configuration of three cylinders in a row cooled by a slot jet of air. ASME, HTD (Pub), Fund. Single-Phase Conv. Heat Tr, HTD 366, 39-44.

Gori, F., Bossi, L., 2002. Cooling of two cylinders in a row by a slot jet of air. Int. J. Transp. Phenom. 4, 245-256.

Gori, F., Bossi, L., 2003. Optimal slot height in the jet cooling of a circular cylinder. Appl. Therm. Eng. 23 (7), 859-870.

Gori, F., Coppa, P., 1998. Circumferential variation of heat transfer on three circular cylinders cooled by a slot jet of air. Int. J. Heat Technol. 16 (2), 63-69.

Gori, F., Nino, E., 2003. Fluid dynamics measurements and flow visualizations of a free slot jet of air. ASME, FED (Pub), FED 259, 187-192.

Gori, F., Petracci, I., 2003a. Local heat transfer on a circular cylinder impinged by a submerged slot jet of air. In: Eurotherm Seminar 74, March 23-26, pp. 63-69.

Gori, F., Petracci, I., 2003b. Fluid dynamics measurements in a submerged free slot jet of air. Proc. 14th Int. Symp. Tr. Phen., Bali Indonesia, 6-10th July, pp. 491496.

Gori, F., Petracci, I., 2003c. Fluid dynamics measurements and numerical simulations around a circular cylinder impinged by a submerged slot jet of air. ASME, FED (Pub), FED 259, 179-185.

Gori, F., Petracci, I., 2003d. Heat transfer measurements and numerical simulations in the cooling of a circular cylinder by a slot jet of air. ASME, HTD (Pub), HTD 374 (1), 3-9.

Gori, F., Petracci, I., 2012. Influence of turbulence on heat transfer upon a cylinder impinged by a slot jet of air. Appl. Therm. Eng. 49, 106-117.

Gori, F., Petracci, I., 2013. On the effect of the slot height in the cooling of a circula cylinder with a rectangular jet. Int. Commun. Heat Mass Transfer 48, 8-14.

Gori, F., Petracci, I., 2014. Influence of screen solidity ratio on heat transfer upon a cylinder impinged by a rectangular jet. Int. J. Heat Mass Transfer, http:// dx.doi.org/10.1016/j.ijheatmasstransfer.2014.10.004.

Gori, F., De Nigris, F., Nino, E., 2002a. Fluid dynamics measurements and optica visualization of the evolution of a submerged slot jet of air. Proc. 12th Int. Heat Tr. Conf., vol. 2. Elsevier, Grenoble, France, pp. 303-308.

Gori, F., De Nigris, F., Pippione, E., Scavarda, G., 2002b. Cooling of finned cylinders by a jet flow of air. ASME, PID (Pub), PID 7, 117-122.
Gori, F., Borgia, M., Doro Altan, A., 2003a. Cooling of a finned cylinder by a jet flow of air. ASME, PID (Pub), PID 8, 147-152.

Gori, F., Pacchiarotti, G., Petracci, I., 2003b. Pulsating jets cooling circular cylinders. ASME, PID (Pub), PID 8, 153-159.

Gori, F., Borgia, M., Doro Altan, A., Mascia, M., Petracci, I., 2005. Cooling of a finned cylinder by a jet flow of air. ASME J. Heat Trans. 127 (12), 1416-1421.

Gori, F., Nino, E., Petracci, I., 2007a. Shadowgraph visualizations of a submerged free slot jet of air. Int. J. Heat Technol. 25 (1), 157-164.

Gori, F., Petracci, I., Tedesco, V., 2007b. Cooling of two smooth cylinders in row by a slot jet of air with low turbulence. Appl. Therm. Eng. 27 (14-15), 2415-2425.

Gori, F., Mascia, M., Petracci, I., 2011. Air cooling of a finned cylinder with slot jets of different height. Int. J. Therm. Sci. 50, 1583-1593.

Gori, F., Angelino, M., Boghi, A., Petracci, I., 2012a. Preliminary numerical solutions of the evolution of free jets. In: ASME International Mechanical Engineering Conference and Exposition, Proceedings (IMECE), 7 (D), pp. 463-469.

Gori, F., Petracci, I., Angelino, M., 2012b. Experimental and numerical heat transfer on a cylinder cooled by two rectangular jets of different heights. ASME, IMECE, 7 (D), pp. 2679-2688.

Gori, F., Petracci, I., Angelino, M., 2013. Flow evolution of a turbulent submerged two-dimensional rectangular free jet of air. Average particle image velocimetry (PIV) visualizations and measurements. Int. J. Heat Fluid Flow 44, 764-775.

Gutmark, E., Wygnanski, I., 1976. The planar turbulent jet. J. Fluid Mech. 73 (3), 465-495.

Hsiao, F.-B., Lim, Y.-C., Huang, J.-M., 2010. On the near-field flow structure and mode behaviors for the right-angle and sharp-edged orifice plane jet. Exp. Thermal Fluid Sci. 34, 1282-1289.

Keane, R.D., Adrian, R.J., 1990. Optimization of particle image velocimeters. Part I: Double pulsed systems. Meas. Sci. Tech. 1, 1202-1215.

Moffat, R.J., 1988. Describing the uncertainties in experimental results. Exp. Thermal Fluid Sci., 3-17.

Potho, S., Longmire, E.K., 2001. Asymmetric forcing of a turbulent rectangular jet with a piezometric actuator. Phys. Fluids 13 (5), 1480-1491.

van der Hegge Zijnen, B.G., 1958. Measurements of the velocity distribution in a plane turbulent jet of air. Appl. Sci. Res. A 7, 257-276.

van Dyke, M., 1982. An Album of Fluid Motion. The Parabolic Press, Stanford, California, USA. 